UCB-PTH-09/05

LBNL-1936E

CERN-PH-TH/2009-001

SU-ITP-09/04

\title{
Duality, Entropy and ADM Mass in Supergravity
}

\author{
Bianca L. Cerchiai ${ }^{\boldsymbol{\phi}, \diamond}$, Sergio Ferrara ${ }^{\boldsymbol{k}, b}$, \\ Alessio Marrani ${ }^{\ominus, \downarrow}$ and Bruno Zumino ${ }^{\infty} \diamond$
}

\author{
- Lawrence Berkeley National Laboratory, \\ Theory Group, Bldg 50A5104 \\ 1 Cyclotron Rd, Berkeley, CA 94720-8162, USA \\ BLCerchiai@lbl.gov, zumino@thsrv.lbl.gov \\ $\diamond$ Department of Physics, University of California, \\ Berkeley, CA 94720-8162, USA \\ \&. Theory division, CERN, Geneva, Switzerland \\ CH 1211, Geneva 23, Switzerland \\ sergio.ferrara@cern.ch \\ b INFN - LNF, \\ Via Enrico Fermi 40, I-00044 Frascati, Italy \\ marrani@lnf. infn.it \\ $\odot$ Stanford Institute for Theoretical Physics \\ Department of Physics, 382 Via Pueblo Mall, Varian Lab, \\ Stanford University, Stanford, CA 94305-4060, USA
}

\begin{abstract}
We consider the Bekenstein-Hawking entropy-area formula in four dimensional extended ungauged supergravity and its electric-magnetic duality property.

Symmetries of both "large" and "small" extremal black holes are considered, as well as the ADM mass formula for $\mathcal{N}=4$ and $\mathcal{N}=8$ supergravity, preserving different fraction of supersymmetry.

The interplay between BPS conditions and duality properties is an important aspect of this investigation.
\end{abstract}




\section{Contents}

1 Introduction 1

2 Electric-Magnetic Duality in Supergravity : Basic Facts 3

$3 \mathcal{N}=8$

$4 \mathcal{N}=4$

$\mathbf{5} \mathcal{N}=2+\underline{\mathbf{2 6}}$

6 ADM Mass for BPS Extremal Black Hole States 35

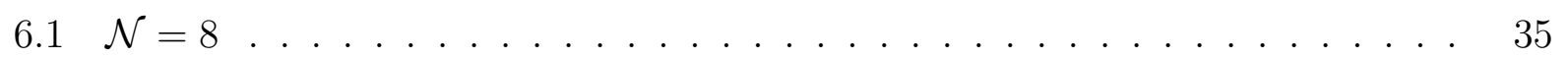

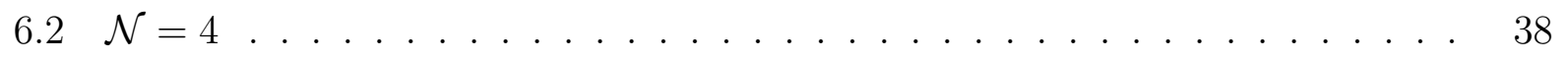

\section{Introduction}

In $d=4$ extended ungauged supergravity theories based on scalar manifolds which are (at least locally) symmetric spaces

$$
M=\frac{G}{H},
$$

it is known that the classification of static, spherically symmetric and asymptotically flat extremal black hole $(\mathrm{BH})$ solutions is made in terms of charge orbits of the corresponding classical electric-magnetic duality group group $G$ [1, 2, 3, 4, 5, 6] (later called $U$-duality $\left.\right|^{1}$ in string theory) .

These orbits correspond to certain values taken by a duality invariant $2^{2}$ combination of the "dressed" central charges and matter charges. Denoting such an invariant by $\mathcal{I}$, the set of scalars parametrizing the symmetric manifold $M$ by $\phi$, and the set of "bare" magnetic and electric charges of the (dyonic) $\mathrm{BH}$ configuration by the $2 n \times 1$ symplectic vector

$$
\mathcal{P} \equiv\left(\begin{array}{c}
p^{\Lambda} \\
q_{\Lambda}
\end{array}\right), \Lambda=1, \ldots, n,
$$

then it holds that

$$
\partial_{\phi} \mathcal{I}(\phi, \mathcal{P})=0 \Leftrightarrow \mathcal{I}=\mathcal{I}(\mathcal{P})
$$

\footnotetext{
${ }^{1}$ Here $U$-duality is referred to as the "continuous" version, valid for large values of the charges, of the $U$-duality groups introduced by Hull and Townsend [7].

${ }^{2}$ By duality invariant, throughout our treatment we mean that such a combination is $G$-invariant. Thus, it is actually independent on the scalar fields, and it depends only on "bare" electric and magnetic (asymptotical) charges (defined in Eq. (1.2)).
} 
In some cases, the relevant invariant $\mathcal{I}$ is not enough to characterize the orbit, and additional constraints are needed. This is especially the case for the so-called ${ }^{3}$ "small" BHs, in which case $\mathcal{I}=0$ on the corresponding orbit [3, 4, 9].

An explicit expression for the $E_{7(7)}$-invariant [10] was firstly introduced in supergravity in [11, and then adopted in the study of $\mathrm{BH}$ entropy in [12]. The additional $U$-invariant constraints which specify charge orbits with higher supersymmetry were given in [3]. The corresponding (large and small) charge orbits for $\mathcal{N}=8$ and exceptional $\mathcal{N}=2$ supergravity were determined in [4], whereas the large orbits for all other symmetric $\mathcal{N}=2$ supergravities were obtained in [6], and then in [13] for all $\mathcal{N}>2$-extended theories. Furthermore, the invariant for $\mathcal{N}=4$ supergravity was earlier discussed in [14, 15].

The invariants play an important role in the attractor mechanism [16, 17, 18, 19, 20, because the Bekenstein-Hawking BH entropy [8], determined by evaluating the effective black hole potential ([18, 19, 20])

$$
V_{B H}(\phi, \mathcal{P}) \equiv-\frac{1}{2} \mathcal{P}^{T} \mathcal{M}(\phi) \mathcal{P}
$$

at its critical points, actually coincides with the relevant invariant:

$$
\frac{S_{B H}}{\pi}=\left.V_{B H}\right|_{\partial_{\phi} V_{B H}=0}=V_{B H}\left(\phi_{H}(\mathcal{P}), \mathcal{P}\right)=|\mathcal{I}(\mathcal{P})|^{1 / 2}(\text { or }|\mathcal{I}(\mathcal{P})|) .
$$

In Eq. (1.4) $\mathcal{M}$ stands for the $2 n \times 2 n$ real (negative definite) symmetric scalar-dependent symplectic matrix

$$
\mathcal{M}(\phi) \equiv\left(\begin{array}{cc}
\operatorname{Im}_{\mathcal{N}} \mathcal{N}_{\Lambda \Sigma}+\operatorname{Re}_{\mathcal{N}}(\operatorname{Im} \mathcal{N})^{-1 \mid \Xi \Delta} \operatorname{ReN}_{\Delta \Sigma} & -\operatorname{Re}_{\Lambda} \mathcal{N}_{\Lambda \Xi}(\operatorname{Im} \mathcal{N})^{-1 \mid \Xi \Sigma} \\
-(\operatorname{Im} \mathcal{N})^{-1 \mid \Lambda \Delta} \operatorname{ReN}_{\Xi \Sigma} & (\operatorname{Im} \mathcal{N})^{-1 \mid \Lambda \Sigma}
\end{array}\right)
$$

defined in terms of the normalization of the Maxwell and topological terms

$$
\operatorname{Im} \mathcal{N}_{\Lambda \Sigma}(\phi) F^{\Lambda} F^{\Sigma}, \quad \operatorname{Re} \mathcal{N}_{\Lambda \Sigma}(\phi) F^{\Lambda} \tilde{F}^{\Sigma}
$$

of the corresponding supergravity theory (see e.g. [21, 22] and Refs. therein). Furthermore, in Eq. (1.5) $\phi_{H}(\mathcal{P})$ denotes the set of charge-dependent, stabilized horizon values of the scalars, solutions of the criticality conditions for $V_{B H}$ :

$$
\left.\frac{\partial V_{B H}(\phi, \mathcal{P})}{\partial \phi}\right|_{\phi=\phi_{H}(\mathcal{P})} \equiv 0
$$

For the case of charge orbits corresponding to small BHs, in the case of a single-center solution $\mathcal{I}(\mathcal{P})=0$, and thus the event horizon area vanishes, and the solution is singular

\footnotetext{
${ }^{3}$ Throughout the present treatment, we will respectively call small or large (extremal) BHs those BHs with vanishing or non-vanishing area of the event horizon (and therefore with vanishing or non-vanishing Bekenstein-Hawking entropy [8]). For symmetric geometries, they can be $G$-invariantly characterized respectively by $\mathcal{I}=0$ or by $\mathcal{I} \neq 0$.

${ }^{4}$ Attention should be paid in order to distinguish between the notations of the number $\mathcal{N}$ of supercharges of a supergravity theory and the kinetic vector matrix $\mathcal{N}_{\Lambda \Sigma}$ introduced in Eqs. (1.6) and (1.7).
} 
(i.e. with vanishing Bekenstein-Hawking entropy). However, the charge orbits with vanishing duality invariant play a role for multi-center solutions as well as for elementary BH constituents through which large (i.e. with non-vanishing Bekenstein-Hawking entropy) BHs are made [23, 24, 25].

In the present investigation, we re-examine the duality invariant and the $U$-invariant classification of charge orbits of $\mathcal{N}=8, d=4$ supergravity, we give a complete analysis of the $\mathcal{N}=4$ large and small charge orbits, and we also derive a diffeomorphism-invariant expression of the $\mathcal{N}=2$ duality invariant, which is common to all symmetric spaces and which is completely independent on the choice of a symplectic basis.

The paper is organized as follows.

In Sect. 2 we recall some basic facts about electric-magnetic duality in $\mathcal{N}$-extended supergravity theories, firstly treated in [2]. The treatment follows from the general analysis of [1], and the dictionary between that paper and the present work is given.

In Sect. 3 we re-examine $\mathcal{N}=8, d=4$ supergravity and the $E_{7(7)}$-invariant characterization of its charge orbits. This refines, re-organizes and extends the various results of [3, 4, 5, 9].

In Sect. 4 we reconsider matter coupled $\mathcal{N}=4, d=4$ supergravity. The $S L(2, \mathbb{R}) \times$ $S O(6, M)$-invariant characterization of all its BPS and non-BPS charge orbits, firstly obtained in [3, 9], is the starting point of the novel results presented in this Section.

Sect. 5 is devoted to the analysis of the $\mathcal{N}=2, d=4$ case [3]. Beside the generalities on the special Kähler geometry of Abelian vector multiplets' scalar manifold, the results of this Section are novel. In particular, a formula for the duality invariant is determined, which is diffeomorphism-invariant and holds true for all symmetric special Kähler manifolds (see e.g. [26] and Refs. therein), regardless of the considered symplectic basis.

Sect. 6, starting from the analysis of [3, 9], deals with the issue of the ADM mass [27] in $\mathcal{N}=8$ (Subsect. 6.1) and $\mathcal{N}=4$ (Subsect. 6.2), ungauged $d=4$ supergravities. In general, for all supersymmetric orbits the $A D M$ mass has a known explicit expression, depending on the number of supersymmetries preserved by the state which is supported by the considered orbit (saturating the BPS [28] bound).

\section{Electric-Magnetic Duality in Supergravity : Basic Facts}

The basic requirement for consistent coupling of a non-linear sigma model based on a symmetric manifold (1.1) to $\mathcal{N}$-extended, $d=4$ supergravity (see e.g. [21] and Refs. therein) is that the vector field strengths and their duals (through Legendre transform with respect the Lagrangian density $\mathcal{L}$ )

$$
F^{\Lambda}, \quad G_{\Lambda} \equiv \frac{\delta \mathcal{L}}{\delta F^{\Lambda}},
$$


belong to a symplectic representation $\mathbf{R}_{s}$ of the global (classical, see Footnote 1 ) $U$-duality group $G$, given by $2 n \times 2 n$ matrices with block structure

$$
\left(\begin{array}{ll}
A & B \\
C & D
\end{array}\right) \in S p(2 n, \mathbb{R})
$$

where $A, B, C$ and $D$ are $n \times n$ real matrices. By defining the $2 n \times 2 n$ symplectic metric (each block being $n \times n$ )

$$
\Omega \equiv\left(\begin{array}{cc}
0 & -1 \\
1 & 0
\end{array}\right)
$$

the finite symplecticity condition for a $2 n \times 2 n$ real matrix $P$

$$
P^{T} \Omega P=\Omega
$$

yields the following relations to hold for the block components of the matrix defined in Eq. (2.2):

$$
\begin{aligned}
A^{T} C-C^{T} A & =0 \\
B^{T} D-D^{T} B & =0 \\
A^{T} D-C^{T} B & =1 .
\end{aligned}
$$

An analogous, equivalent definition of the representation $\mathbf{R}_{s}$ is the following one: $\mathbf{R}_{s}$ is real and it contains the singlet in its 2-fold antisymmetric tensor product

$$
\left(\mathbf{R}_{s} \times \mathbf{R}_{s}\right)_{a} \ni \mathbf{1}
$$

If the basic requirements (2.5)-(2.7) or (2.8) are met, the coset representative of $M$ in the symplectic representation $\mathbf{R}_{s}$ is given by the (scalar-dependent) $2 n \times 2 n$ matrix

$$
S(\phi) \equiv\left(\begin{array}{cc}
A(\phi) & B(\phi) \\
C(\phi) & D(\phi)
\end{array}\right) \in S p(2 n, \mathbb{R}) .
$$

A particular role is played by the two (scalar-dependent) complex $n \times n$ matrices $f$ and $h$, which do satisfy the properties

$$
\begin{aligned}
-f^{\dagger} h+h^{\dagger} f & =i 1, \\
-f^{T} h+h^{T} f & =0 .
\end{aligned}
$$

The constraining relations 2.10 and 2.11) are equivalent to require that

$$
S(\phi)=\sqrt{2}\left(\begin{array}{cc}
\operatorname{Ref} & -\operatorname{Imf} \\
\operatorname{Reh} & -\operatorname{Imh}
\end{array}\right),
$$


or equivalently:

$$
\begin{aligned}
f & =\frac{1}{\sqrt{2}}(A-i B) ; \\
h & =\frac{1}{\sqrt{2}}(C-i D) .
\end{aligned}
$$

In order to make contact with the formalism introduced by Gaillard and Zumino in [1, it is convenient to use another (complex) basis, namely the one which maps an element $S \in S p(2 n, \mathbb{R})$ into an element $U \in U(n, n) \cap S p(2 n, \mathbb{C})$. The change of basis is exploited through the matrix

$$
\mathcal{A} \equiv \frac{1}{\sqrt{2}}\left(\begin{array}{cc}
1 & 1 \\
-i 1 & i 1
\end{array}\right), \quad \mathcal{A}^{-1}=\mathcal{A}^{\dagger} .
$$

The (scalar-dependent) matrix $U$ is thus defined as follows:

$$
U(\phi) \equiv \mathcal{A}^{-1} S \mathcal{A}=\frac{1}{\sqrt{2}}\left(\begin{array}{ll}
f+i h & \bar{f}+i \bar{h} \\
f-i h & \bar{f}-i \bar{h}
\end{array}\right) \in U(n, n) \cap S p(2 n, \mathbb{C}) .
$$

This is the matrix named $S$ in Eq. (5.1) of [1]. Correspondingly, the $S p(2 n, \mathbb{R})$-covariant vector $\left(F^{\Lambda}, G_{\Lambda}\right)^{T}$ is mapped into the vector

$$
\mathcal{A}^{-1}\left(\begin{array}{c}
F^{\Lambda} \\
G_{\Lambda}
\end{array}\right)=\frac{1}{\sqrt{2}}\left(\begin{array}{cc}
1 & i 1 \\
1 & -i 1
\end{array}\right)\left(\begin{array}{c}
F^{\Lambda} \\
G_{\Lambda}
\end{array}\right)=\frac{1}{\sqrt{2}}\left(\begin{array}{c}
F^{\Lambda}+i G_{\Lambda} \\
F^{\Lambda}-i G_{\Lambda}
\end{array}\right) .
$$

The kinetic vector matrix $\mathcal{N}_{\Lambda \Sigma}$ appearing in Eqs. (1.6) and (1.7) is given by (in matrix notation)

$$
\mathcal{N}(\phi)=h f^{-1}=\left(f^{-1}\right)^{T} h^{T},
$$

and it is named $-i \bar{K}$ in [1].

Thus, by introducing the $2 n \times 1(n \times n$ matrix-valued $)$ complex vector

$$
\Xi \equiv\left(\begin{array}{c}
f \\
h
\end{array}\right)
$$

and recalling the definition $(1.6)$, the matrix $\mathcal{M}$ can be written as

$$
\begin{aligned}
& \mathcal{M}(\phi)=-i \Omega+2 \Omega \Xi(\Omega \Xi)^{\dagger}=-i \Omega-2 \Omega \Xi \Xi^{\dagger} \Omega= \\
& =-i \Omega-2\left(\begin{array}{c}
-h \\
f
\end{array}\right)\left(\begin{array}{ll}
h^{\dagger}, & -f^{\dagger}
\end{array}\right)= \\
& =-i\left(\begin{array}{cc}
0 & -1 \\
1 & 0
\end{array}\right)+2\left(\begin{array}{cc}
h h^{\dagger} & -h f^{\dagger} \\
-f h^{\dagger} & f f^{\dagger}
\end{array}\right) \text {. }
\end{aligned}
$$


Eqs. 1.4, (1.6) and (2.20) imply that

$$
\begin{aligned}
V_{B H}(\phi, \mathcal{P}) & \equiv-\frac{1}{2} \mathcal{P}^{T} \mathcal{M}(\phi) \mathcal{P}=\operatorname{Tr}\left(\mathcal{Z} \mathcal{Z}^{\dagger}\right)=\operatorname{Tr}\left(\mathcal{Z}^{\dagger} \mathcal{Z}\right)= \\
& =\sum_{A>B=1}^{\mathcal{N}} Z_{A B} \bar{Z}^{A B}+Z_{I} \bar{Z}^{I}=\frac{1}{2} Z_{A B} \bar{Z}^{A B}+Z_{I} \bar{Z}^{I}= \\
& =\frac{1}{2} \operatorname{Tr}\left(Z Z^{\dagger}\right)+Z_{I} \bar{Z}^{I}=\frac{1}{2} \operatorname{Tr}\left(Z^{\dagger} Z\right)+Z_{I} \bar{Z}^{I},
\end{aligned}
$$

where $(A, B=1, \ldots, \mathcal{N}$ and $I=1, \ldots, m$ throughout; recall $\Lambda=1, \ldots, n)$

$$
\begin{aligned}
& \mathcal{Z} \equiv \mathcal{P}^{T} \Omega \Xi=q f-p h=\left(Z_{A B}(\phi, \mathcal{P}), Z_{I}(\phi, \mathcal{P})\right) \\
& \Uparrow \\
& \mathcal{Z}^{\dagger} \equiv-\Xi^{\dagger} \Omega \mathcal{P}=f^{\dagger} q-h^{\dagger} p=\left(\begin{array}{c}
\bar{Z}^{A B}(\phi, \mathcal{P}) \\
\bar{Z}^{I}(\phi, \mathcal{P})
\end{array}\right) \\
& Z_{A B}(\phi, \mathcal{P}) \equiv f_{A B}^{\Lambda} q_{\Lambda}-h_{A B \mid \Lambda} p^{\Lambda} \\
& Z_{I}(\phi, \mathcal{P}) \equiv \bar{f}_{I}^{\Lambda} q_{\Lambda}-\bar{h}_{I \mid \Lambda} p^{\Lambda}
\end{aligned}
$$

Thus, Eq. (2.21) yields the "BH potential" $V_{B H}(\phi, \mathcal{P})$ to be nothing but the sum of the squares of the "dressed" charges. It is here worth noticing that $\left(f_{A B}^{\Lambda}, \bar{f}_{I}^{\Lambda}\right)$ and $\left(h_{A B \mid \Lambda}, \bar{h}_{I \mid \Lambda}\right)$ are $n \times n$ complex matrices, because it holds that ${ }^{5} f_{A B}^{\Lambda}=f_{[A B]}^{\Lambda}, h_{A B \mid \Lambda}=h_{[A B] \mid \Lambda}$ (thus implying $Z_{A B}=Z_{[A B]}$, and

$$
n=\frac{\mathcal{N}(\mathcal{N}-1)}{2}+m
$$

where $\mathcal{N}$ stands for the number of spinorial supercharges (see Footnote 4 ), and $m$ denotes the number of matter multiplets coupled to the supergravity multiplet, except for $\mathcal{N}=6$, $d=4$ pure supergravity, for which $m=1$.

Eqs. (2.24) and (2.25) are the basic relation between the (scalar-dependent) "dressed" charges $Z_{A B}$ and $Z_{I}$ and the (scalar-independent) "bare" charges $\mathcal{P}$. It is worth remarking that $Z_{A B}$ is the "central charge matrix function", whose asymptotical value appears in the right-hand side of the $\mathcal{N}$-extended $(d=4)$ supersymmetry algebra, pertaining to the asymptotical Minkowski space-time background:

$$
\left\{Q_{\alpha}^{A}, Q_{\beta}^{B}\right\}=\epsilon_{\alpha \beta} Z^{A B}\left(\phi_{\infty}, \mathcal{P}\right),
$$

where $\phi_{\infty}$ denotes the set of values taken by the scalar fields at radial infinity $(r \rightarrow \infty)$ within the considered static, spherically symmetric and asymptotically flat dyonic extremal BH background. Notice that the indices $A, B$ of the central charge matrix are raised and lowered

\footnotetext{
${ }^{5}$ Unless otherwise noted, square brackets denote antisymmetrization with respect to the enclosed indices.
} 
with the metric of the relevant $\mathcal{R}$-symmetry group of the corresponding supersymmetry algebra.

By denoting the ADM mass [27] of the considered BH background by $M_{A D M}\left(\phi_{\infty}, \mathcal{P}\right)$, the BPS bound [28] implies that

$$
M_{A D M}\left(\phi_{\infty}, \mathcal{P}\right) \geqslant\left|\mathbf{Z}_{1}\left(\phi_{\infty}, \mathcal{P}\right)\right| \geqslant \ldots \geqslant\left|\mathbf{Z}_{[\mathcal{N} / 2]}\left(\phi_{\infty}, \mathcal{P}\right)\right|
$$

where $\mathbf{Z}_{1}(\phi, \mathcal{P}), \ldots, \mathbf{Z}_{[\mathcal{N} / 2]}(\phi, \mathcal{P})$ denote the set of skew-eigenvalues of $Z_{A B}(\phi, \mathcal{P})$, and here square brackets denote the integer part of the enclosed number. If $1 \leqslant \mathbf{k} \leqslant[\mathcal{N} / 2]$ of the bounds expressed by Eq. (2.28) are saturated, the corresponding extremal $\mathrm{BH}$ state is named to be $\frac{\mathbf{k}}{\mathcal{N}}$-BPS. Thus, the minimal fraction of total supersymmetries (pertaining to the asymptotically flat space-time metric) preserved by the extremal BH background within the considered assumptions is $\frac{1}{\mathcal{N}}$ (for $\mathbf{k}=1$ ), while the maximal one is $\frac{1}{2}$ (for $\mathbf{k}=\frac{\mathcal{N}}{2}$ ). See Sect. 6 for further details.

We end the present Section with some considerations on the issue of duality invariants.

A duality invariant $\mathcal{I}$ is a suitable linear combination (in general with complex coefficients) of ( $\phi$-dependent) $H$-invariant combinations of $Z_{A B}(\phi, \mathcal{P})$ and $Z_{I}(\phi, \mathcal{P})$ such that Eq. (1.3) holds, i.e. such that $\mathcal{I}$ is invariant under $G$, and thus $\phi$-independent:

$$
\mathcal{I}=\mathcal{I}\left(Z_{A B}(\phi, \mathcal{P}), Z_{I}(\phi, \mathcal{P})\right)=\mathcal{I}(\mathcal{P})
$$

In presence of matter coupling, a charge configuration $\mathcal{P}$ (and thus a certain orbit of the symplectic representation of the $U$-duality group $G$, to which $\mathcal{P}$ belongs) is called supersymmetric iff, by suitably specifying $\phi=\phi(\mathcal{P})$, it holds that

$$
Z_{I}(\phi(\mathcal{P}), \mathcal{P})=0, \forall I=1, \ldots, m .
$$

Notice that the conditions 2.30 cannot hold identically in $\phi$, otherwise such conditions would be $G$-invariant, which generally are not. Indeed, in order for the supersymmetry constraints 2.30 to be invariant (or covariant) under $G$, the following conditions must hold identically in $\phi$ :

$$
\partial_{\phi} Z_{I}(\phi, \mathcal{P})=0, \quad \forall \phi \in M
$$

Therefore, supersymmetry conditions are not generally $G$-invariant (i.e. U-invariant), otherwise extremal BH attractors (which are large) supported by supersymmetric charge configurations would not exist.

Nevertheless, in some supergravities it is possible to give $U$-invariant supersymmetry conditions. In light of previous reasoning, such $U$-invariant supersymmetric conditions cannot stabilize the scalar fields in terms of charges (by implementing the attractor mechanism in the considered framework), because such $U$-invariant conditions are actually identities, and not equations, for the set of scalar fields $\phi$. Actually, $U$-invariant supersymmetry conditions can be given for all supersymmetric charge orbits supporting small BHs (for which the classical attractor mechanism does not hold). This can be seen e.g. in $\mathcal{N}=8$ (pure) and $\mathcal{N}=4$ (matter coupled) $d=4$ supergravities, respectively treated in Sects. 3 and 4 . 


\section{$3 \mathcal{N}=8$}

The scalar manifold of the maximal, namely $\mathcal{N}=8$, supergravity in $d=4$ is the symmetric real coset

$$
\left(\frac{G}{H}\right)_{\mathcal{N}=8, d=4}=\frac{E_{7(7)}}{S U(8)}, \operatorname{dim}_{\mathbb{R}}=70,
$$

where the usual notation for non-compact forms of exceptional Lie groups is used, with subscripts denoting the difference "\# non-compact generators - \# compact generators". This theory is pure, i.e. matter coupling is not allowed. The classical (see Footnote 1) $U$ duality group is $E_{7(7)}$. Moreover, the $\mathcal{R}$-symmetry group is $S U(8)$ and, due to the absence of matter multiplets, it is nothing but the stabilizer of the scalar manifold (3.1) itself.

The Abelian vector field strengths and their duals, as well the corresponding fluxes (charges), sit in the fundamental representation $\mathbf{5 6}$ of the global, classical $U$-duality group $E_{7(7)}$. Such a representation determines the embedding of $E_{7(7)}$ into the symplectic group $S p(56, \mathbb{R})$, which is the largest symmetry acting linearly on charges. The $\mathbf{5 6}$ of $E_{7(7)}$ admits an unique invariant, which will be denoted by $\mathcal{I}_{4, \mathcal{N}=8}$ throughout. $\mathcal{I}_{4, \mathcal{N}=8}$ is quartic in charges, and it was firstly determined in [11].

More precisely, $\mathcal{I}_{4, \mathcal{N}=8}$ is the unique combination of $Z_{A B}(\phi, \mathcal{P})$ satisfying

$$
\partial_{\phi} \mathcal{I}_{4, \mathcal{N}=8}\left(Z_{A B}(\phi, \mathcal{P})\right)=0, \quad \forall \phi \in \frac{E_{7(7)}}{S U(8)} .
$$

Eq. 3.2 can be computed by using the Maurer-Cartan Eqs. of the coset $\frac{E_{7(7)}}{S U(8)}$ (see e.g. [29] and Refs. therein):

$$
\nabla Z_{A B}=\frac{1}{2} P_{A B C D} \bar{Z}^{C D}
$$

or equivalently by performing an infinitesimal $\frac{E_{7(7)}}{S U(8)}$-transformation of the central charge matrix (see e.g. [29] and Refs. therein):

$$
\delta_{\xi_{A B C D}} Z_{A B}=\frac{1}{2} \xi_{A B C D} \bar{Z}^{C D},
$$

where $\nabla$ and $P_{A B C D}$ respectively denote the covariant differential operator and the Vielbein 1-form in $\frac{E_{7(7)}}{S U(8)}$, and the infinitesimal $\frac{E_{7(7)}}{S U(8)}$-parameters $\xi_{A B C D}$ satisfy the reality constraint

$$
\xi_{A B C D}=\frac{1}{4 !} \epsilon_{A B C D E F G H} \bar{\xi}^{E F G H} .
$$

As firstly found in [11] and rigorously re-obtained in [29], the unique solution of Eq. (3.2) reads:

$$
\mathcal{I}_{4, \mathcal{N}=8}=\frac{1}{2^{2}}\left[2^{2} \operatorname{Tr}\left(\left(Z_{A C} \bar{Z}^{B C}\right)^{2}\right)-\left(\operatorname{Tr}\left(Z_{A C} \bar{Z}^{B C}\right)\right)^{2}+2^{5} \operatorname{Re}\left(\operatorname{Pf}\left(Z_{A B}\right)\right)\right],
$$

where the Pfaffian of $Z_{A B}$ is defined as [11]

$$
\operatorname{Pf}\left(Z_{A B}\right) \equiv \frac{1}{2^{4} 4 !} \epsilon^{A B C D E F G H} Z_{A B} Z_{C D} Z_{E F} Z_{G H},
$$


and it holds that (see e.g. [29])

$$
\left|\operatorname{Pf}\left(Z_{A B}\right)\right|=\left|\operatorname{det}\left(Z_{A B}\right)\right|^{1 / 2} .
$$

In 29] it was indeed shown that, although each of the three terms of the expression (3.6) is $S U$ (8)-invariant but scalar-dependent, only the combination given by the expression (3.6) is actually $E_{7(7)}$-independent and thus scalar-independent, satisfying

$$
\delta_{\xi_{A B C D}} \mathcal{I}_{4, \mathcal{N}=8}=0
$$

with Eqs. (3.4) and (3.5) holding true.

It is here worth commenting a bit further about formula (3.6). The first two terms in its right-hand side are actually $U(8)$-invariant, while the third one, namely $2^{5} \operatorname{Re}\left(\operatorname{Pf}\left(Z_{A B}\right)\right)$, is only $S U(8)$-invariant. Such a third term introduces an $S U(8)$-invariant phase $\varphi_{Z}$, defined as (one fourth of) the overall phase of the central charge matrix, when this latter is reduced to a skew-diagonal form in the so-called normal frame through an $S U$ (8)-transformation:

$$
Z_{A B} \stackrel{S U(8)}{\longrightarrow} Z_{A B, \text { skew-diag. }} \equiv e^{i \varphi_{Z} / 4}\left(\begin{array}{cccc}
e_{1} & & & \\
& e_{2} & & \\
& & e_{3} & \\
& & & e_{4}
\end{array}\right) \otimes \epsilon, \quad e_{i} \in \mathbb{R}^{+}, \forall i=1, \ldots, 4,
$$

where the ordering $e_{1} \geqslant e_{2} \geqslant e_{3} \geqslant e_{4}$ can be performed without any loss of generality, and the $2 \times 2$ symplectic metric

$$
\epsilon \equiv\left(\begin{array}{cc}
0 & -1 \\
1 & 0
\end{array}\right)
$$

has been introduced (notice $\epsilon=\Omega$ for $n=1$, as defined in Eq. (2.3)). For non-vanishing (in general all different) skew-eigenvalues $e_{i}$, the symmetry group of $Z_{A B, \text { skew-diag. }}$ is $(U S p(2))^{4} \sim$ $(S U(2))^{4}$. Thus, beside the 4 skew-eigenvalues $e_{i}$ and the phase $\varphi_{Z}$, the generic $Z_{A B}$ is described by $51=\operatorname{dim}_{\mathbb{R}}\left(\frac{S U(8)}{(S U(2))^{4}}\right)$ "generalized angles". Consistently, the total number of parameters is $4+1+51=56$, which is the real dimension of the fundamental representation 56, defining the embedding of $E_{7(7)}$ into $S p(56, \mathbb{R})$.

Equivalently, $\varphi_{Z}$ can be defined through the Pfaffian of $Z_{A B}$ as follows:

$$
e^{2 i \varphi_{Z}} \equiv \frac{\operatorname{Pf}\left(Z_{A B}\right)}{\operatorname{Pf}\left(\bar{Z}_{A B}\right)}
$$

where clearly $\operatorname{Pf}\left(\bar{Z}_{A B}\right)=\overline{\operatorname{Pf}\left(Z_{A B}\right)}$, as yielded by the definition 3.7$)$. It is then immediate to compute $\varphi_{Z}$ from Eq. (3.6):

$$
\cos \varphi_{Z}(\phi, \mathcal{P})=\frac{\left[2^{2} \mathcal{I}_{4, \mathcal{N}=8}(\mathcal{P})-2^{2} \operatorname{Tr}\left(\left(Z_{A C} \bar{Z}^{B C}\right)^{2}\right)+\left(\operatorname{Tr}\left(Z_{A B} \bar{Z}^{A C}\right)\right)^{2}\right]}{2^{5}\left(\operatorname{det}\left(Z_{A C} \bar{Z}^{B C}\right)\right)^{1 / 4}} .
$$


Notice that through Eq. (3.13) $(\cos ) \varphi_{Z}$ is determined in terms of the scalar fields $\phi$ and of the $\mathrm{BH}$ charges $\mathcal{P}$, also along the small orbits where $\mathcal{I}_{4, \mathcal{N}=8}=0$. However, Eq. 3.13 is not defined in the cases in which $\operatorname{det}\left(Z_{A C} \bar{Z}^{B C}\right)=0$, i.e. when at least one of the eigenvalues of the matrix $Z_{A C} \bar{Z}^{B C}$ vanishes. In such cases, $\varphi_{Z}$ is actually undetermined.

In $\mathcal{N}=8, d=4$ supergravity five distinct orbits of the $\mathbf{5 6}$ of $E_{7(7)}$ exist, as resulting from the analyses performed in [4] and [5]. They can be classified in large and small charge orbits, depending whether they correspond to $\mathcal{I}_{4, \mathcal{N}=8} \neq 0$ or $\mathcal{I}_{4, \mathcal{N}=8}=0$, respectively.

Only two large charge orbits (for which $\mathcal{I}_{4, \mathcal{N}=8} \neq 0$, and the attractor mechanism holds) exist in $\mathcal{N}=8, d=4$ supergravity:

1. The large $\frac{1}{8}$-BPS orbit [4, 5]

$$
\mathcal{O}_{\frac{1}{8}-B P S, \text { large }}=\frac{E_{7(7)}}{E_{6(2)}}, \operatorname{dim}_{\mathbb{R}}=55,
$$

is defined by the $E_{7(7)}$-invariant constraint

$$
\mathcal{I}_{4, \mathcal{N}=8}>0 \text {. }
$$

At the event horizon of the extremal $\mathrm{BH}$, the solution of the $\mathcal{N}=8, d=4$ Attractor Eqs. yields [3, 9, 30]

$$
e_{1} \in \mathbb{R}_{0}^{+}, \quad e_{2}=e_{3}=e_{4}=0,
$$

implying $\operatorname{det}\left(Z_{A B}\right)=0 \Leftrightarrow \operatorname{Pf}\left(Z_{A B}\right)=0$, and thus $\varphi_{Z}$ to be undetermined. Thus, at the event horizon, the symmetry of the skew-diagonalized central charge matrix $Z_{A B, s k e w-d i a g}$. defined in Eq. 3.10 gets enhanced as follows, revealing the maximal compact symmetry of $\mathcal{O}_{\frac{1}{8}-B P S \text {, large }}$ :

$$
(U S p(2))^{4} \stackrel{r \rightarrow r_{H}^{+}}{\longrightarrow} U S p(2) \times S U(6) \sim S U(2) \times S U(6) .
$$

Indeed, $S U(2) \times S U(6)$ is the maximal compact subgroup (mcs, with symmetric embedding [31]) of $E_{6(2)}$ (stabilizer of $\mathcal{O}_{\frac{1}{8}-B P S \text {, large }}$ ) itself.

2. The large non-BPS $\left(Z_{A B} \neq 0\right)$ orbit $[4,5$ ]

$$
\mathcal{O}_{\text {non-BPS, }} \text { AB } \neq 0=\frac{E_{7(7)}}{E_{6(6)}}, \operatorname{dim}_{\mathbb{R}}=55,
$$

is defined by the $E_{7(7)}$-invariant constraint

$$
\mathcal{I}_{4, \mathcal{N}=8}<0 .
$$

At the event horizon of the extremal $\mathrm{BH}$, the solution of the $\mathcal{N}=8, d=4$ Attractor Eqs. yields [3, 9, 30]

$$
e_{1}=e_{2}=e_{3}=e_{4} \in \mathbb{R}_{0}^{+}, \quad \varphi_{Z}=\pi+2 k \pi, k \in \mathbb{Z},
$$


so the skew-eigenvalues of $Z_{A B}$ at the horizon (see Eq. (3.10) ) are complex. Thus, at the event horizon, the symmetry of the skew-diagonalized central charge matrix $Z_{A B, \text { skew-diag. }}$ defined in Eq. (3.10) gets enhanced as follows, revealing the maximal compact symmetry of $\mathcal{O}_{\text {non }-B P S, Z_{A B} \neq 0}$ :

$$
(U S p(2))^{4} \stackrel{r \rightarrow r_{H}^{+}}{\longrightarrow} U S p(8) .
$$

Indeed, $U S p(8)$ is the mcs (with symmetric embedding [31]) of $E_{6(6)}$ (stabilizer of $\left.\mathcal{O}_{\text {non }-B P S, Z_{A B} \neq 0}\right)$ itself.

As mentioned above, for such large charge orbits, corresponding to a non-vanishing quartic $E_{7(7)}$-invariant $\mathcal{I}_{4, \mathcal{N}=8}$ and thus supporting large BHs, the attractor mechanism holds. Consequently, the computations of the Bekenstein-Hawking BH entropy can be performed by solving the criticality conditions for the "BH potential"

$$
V_{B H, \mathcal{N}=8}=\frac{1}{2} Z_{A B} \bar{Z}^{A B}
$$

the result being

$$
\frac{S_{B H,}}{\pi}=\left.V_{B H, \mathcal{N}=8}\right|_{\partial V_{B H, \mathcal{N}=8=0}}=V_{B H, \mathcal{N}=8}\left(\phi_{H}(\mathcal{P}), \mathcal{P}\right)=\left|\mathcal{I}_{4, \mathcal{N}=8}\right|^{1 / 2},
$$

where $\phi_{H}(\mathcal{P})$ denotes the set of solutions to the criticality conditions of $V_{B H, \mathcal{N}=8}$, namely the Attractor Eqs. of $\mathcal{N}=8, d=4$ supergravity:

$$
\partial_{\phi} V_{B H, \mathcal{N}=8}=0, \quad \forall \phi \in \frac{E_{7(7)}}{S U(8)},
$$

expressing the stabilization of the scalar fields purely in terms of supporting charges $\mathcal{P}$ at the event horizon of the extremal BH. Through Eqs. (3.3) and (3.22), Eqs. (3.24) can be rewritten as follows (notice the strict similarity to Eq. (3.40) further below) [30]:

$$
Z_{[A B} Z_{C D]}+\frac{1}{4 !} \epsilon_{A B C D E F G H} \bar{Z}^{E F} \bar{Z}^{G H}=0 .
$$

Actually, the critical potential $\left.V_{B H, \mathcal{N}=8}\right|_{\partial V_{B H, \mathcal{N}=8}=0}$ exhibits some "flat" directions, so not all scalars are stabilized in terms of charges at the event horizon [32, 33]. Thus, Eq. (3.23) yields that the unstabilized scalars, spanning a related moduli space of the considered class of attractor solutions, do not enter in the expression of the BH entropy at all. The moduli spaces $\sqrt{6}$ exhibited by the Attractor Eqs. (3.24)- 3.25) are 33]

$$
\begin{aligned}
\mathcal{M}_{\frac{1}{8}-B P S, \text { large }} & =\frac{E_{6(2)}}{S U(2) \times S U(6)}, \operatorname{dim}_{\mathbb{R}}=40 ; \\
\mathcal{M}_{\text {non-BPS,Z} \text { AB } \neq 0} & =\frac{E_{6(6)}}{U S p(8)}, \operatorname{dim}_{\mathbb{R}}=42 .
\end{aligned}
$$

\footnotetext{
${ }^{6}$ Results obtained by explicit computations within the $\mathcal{N}=2, d=4$ symmetric so-called stu model in [23] and [34] seem to point out that the moduli spaces should be present not only at the event horizon of the considered extremal BH (i.e. for $r \rightarrow r_{H}^{+}$), but also all along the scalar attractor flow $\left(\right.$i.e. $\left.\forall r \geqslant r_{H}\right)$.
} 
As found in [33], the general structure of the moduli spaces of attractor solutions in supergravities based on symmetric scalar manifolds $\frac{G}{H}$ is

$$
\frac{\mathcal{H}_{n c}}{\mathbf{h}}
$$

where $\mathcal{H}_{n c}$ is the non-compact stabilizer of the charge orbit $\frac{G}{\mathcal{H}_{n c}}$ (apart from eventual $U(1)$ factors, $\mathcal{H}_{n c}$ is a non-compact, real form of $\left.H\right)$, and $\mathbf{h}=m c s\left(\mathcal{H}_{n c}\right)$. As justified in

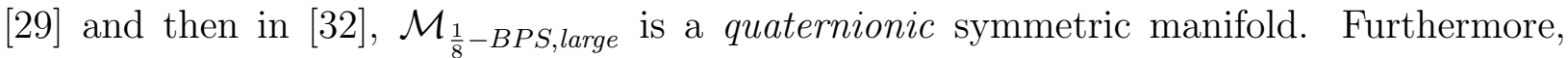
$\mathcal{M}_{n o n-B P S, Z_{A B} \neq 0}$ given by Eq. (3.27) is nothing but the scalar manifold of $\mathcal{N}=8, d=5$ supergravity. The stabilizers of $\mathcal{M}_{\frac{1}{8}-B P S \text {,large }}$ and $\mathcal{M}_{n o n-B P S, Z_{A B} \neq 0}$ exploit the maximal compact symmetry of the corresponding charge orbits; this symmetry becomes fully manifest through the enhancement of the compact symmetry group of $Z_{A B, \text { skew-diag. }}$ at the event horizon of the extremal BH, respectively given by Eqs. (3.17) and (3.21).

It is now convenient to denote with $\lambda_{i}(i=1, \ldots, 4)$ the four real non-negative eigenvalues of the matrix $Z_{A B} \bar{Z}^{C B}=\left(Z Z^{\dagger}\right)_{A}^{C}$. By recalling Eq. 3.10 , one can notice that

$$
\lambda_{i}=e_{i}^{2}
$$

and one can order them as $\lambda_{1} \geqslant \lambda_{2} \geqslant \lambda_{3} \geqslant \lambda_{4}$, without any loss of generality. The explicit expression of $\lambda_{i}$ in terms of $U$ (8)-invariants (namely of $\operatorname{Tr}\left(Z Z^{\dagger}\right), \operatorname{Tr}\left(\left(Z Z^{\dagger}\right)^{2}\right), \operatorname{Tr}\left(\left(Z Z^{\dagger}\right)^{3}\right)$ and $\operatorname{Tr}\left(\left(Z Z^{\dagger}\right)^{4}\right)$, and suitable powers) is given by Eqs. (4.74), (4.75), (4.86) and (4.87) of [9], and it will be used in Sect. 6 to determine the ADM mass for $\frac{\mathbf{k}}{8}$-BPS $(\mathbf{k}=1,2,4)$ extremal BH states.

Three distinct small charge orbits (all with $\mathcal{I}_{4, \mathcal{N}=8}=0$ ) exist, and they all are supersymmetric :

1. The generic small lightlike orbit is $\frac{1}{8}$-BPS, it is defined by the $E_{7(7)}$-invariant constraint

$$
\mathcal{I}_{4, \mathcal{N}=8}=0,
$$

and it reads [4, 5]

$$
\mathcal{O}_{\frac{1}{8}-B P S, \text { small }}=\frac{E_{7(7)}}{F_{4(4)} \times_{s} T_{26}}, \operatorname{dim}_{\mathbb{R}}=55 .
$$

Generally, it yields four different $\lambda_{i}$ 's, and in this case Eq. (3.13) reduces to

$$
\left.\cos \varphi_{Z}(\phi, \mathcal{P})\right|_{\mathcal{I}_{4, \mathcal{N}=8}=0}=-\left.\frac{\left[2^{2} \operatorname{Tr}\left(\left(Z_{A C} \bar{Z}^{B C}\right)^{2}\right)-\left(\operatorname{Tr}\left(Z_{A B} \bar{Z}^{A C}\right)\right)^{2}\right]}{2^{5}\left(\operatorname{det}\left(Z_{A C} \bar{Z}^{B C}\right)\right)^{1 / 4}}\right|_{\mathcal{I}_{4, \mathcal{N}=8}=0} .
$$

In agreement with the results of [4] and [5], the (maximal compact) symmetry of the skew-diagonalized central charge matrix $Z_{A B \text {,skew-diag. }}$ all along the $\frac{1}{8}$-BPS small flow is 
the generic one: $(S U(2))^{4}$. The counting of the parameters of $\mathcal{O}_{\frac{1}{8}-B P S, \text { small }}$ consistently reads: $55=4$ skew-eigenvalues $\lambda_{i}+1$ phase $\varphi_{Z}+51\left(=\operatorname{dim}_{\mathbb{R}}\left(\frac{S U(8)}{(S U(2))^{4}}\right)\right)$ "generalized angles"-1 defining constraint 3.30.

2. The small critical orbit is $\frac{1}{4}$-BPS. It reads [4, 5]

$$
\mathcal{O}_{\frac{1}{4}-B P S}=\frac{E_{7(7)}}{\left(S O(6,5) \times{ }_{s} T_{32}\right) \times T_{1}}, \operatorname{dim}_{\mathbb{R}}=45,
$$

and it is defined by the following differential constraint on $\mathcal{I}_{4, \mathcal{N}=8}[3$, , $]$ :

$$
\frac{\partial \mathcal{I}_{4, \mathcal{N}=8}}{\partial Z_{A B}}=0
$$

which, due to the reality of $\mathcal{I}_{4, \mathcal{N}=8}$, is actually $E_{7(7)}$-invariant. Let us also notice that, due to the homogeneity of $\mathcal{I}_{4, \mathcal{N}=8}$ of degree four in $\mathcal{P}$, Eq. (3.34) implies the constraint 3.30. In particular, along the $\frac{1}{4}$-BPS orbit it holds that (the labelling does not yield any loss of generality)

$$
\lambda_{1}=\lambda_{2}>\lambda_{3}=\lambda_{4} \geqslant 0
$$

If $\operatorname{Pf}\left(Z_{A B}\right) \neq 0$ then

$$
\lambda_{1}=\lambda_{2}>\lambda_{3}=\lambda_{4}>0
$$

and Eq. (3.13) yields $\varphi_{Z}=k \pi, k \in \mathbb{Z}$, so the skew-eigenvalues of $Z_{A B}$ (see Eq. (3.10)

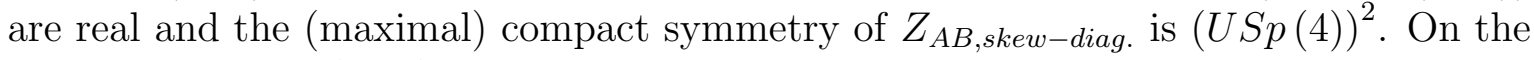
other hand, if $\operatorname{Pf}\left(Z_{A B}\right)=0$ then

$$
\lambda_{1}=\lambda_{2}>\lambda_{3}=\lambda_{4}=0
$$

and $\varphi_{Z}$ is undetermined. In this case, the (maximal compact) symmetry of the skew-

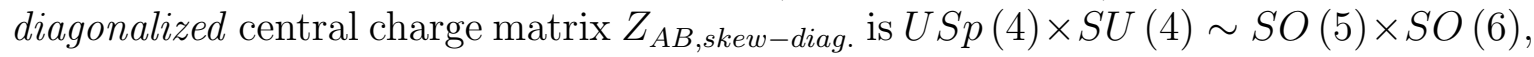
which is the mcs of the non-translational part of the stabilizer of $\mathcal{O}_{\frac{1}{4}-B P S}$, expressing the maximal compact symmetry of $\mathcal{O}_{\frac{1}{4}-B P S}$ itself. In agreement with the results of [4] and [5], the maximal (compact) symmetry of the skew-diagonalized central charge matrix $Z_{A B \text {,skew-diag. }}$ along the $\frac{1}{4}$-BPS small flow (fully manifest in the particular solution (3.37) is $U S p(4) \times S U(4)$. The counting of the parameters of $\mathcal{O}_{\frac{1}{4}-B P S}$ consistently reads: $45=2$ skew-eigenvalues $\lambda_{1}$ and $\lambda_{2}+43\left(=\operatorname{dim}_{\mathbb{R}}\left(\frac{S U(8)}{(U S p(4))^{2}}\right)\right)^{4}$ "generalized angles".

3. The small doubly-critical orbit is $\frac{1}{2}$-BPS, and it reads [4, 5]

$$
\mathcal{O}_{\frac{1}{2}-B P S}=\frac{E_{7(7)}}{E_{6(6)} \times_{s} T_{27}}, \operatorname{dim}_{\mathbb{R}}=28 .
$$

It can be defined in an $E_{7(7)}$-invariant way by performing the following two-step procedure [9]. One starts by considering the requirement that the second derivative of $\mathcal{I}_{4, \mathcal{N}=8}$ 
(with respect to $\left.Z_{A B}\right)$ projected along the adjoint representation $\mathbf{A d j}(S U(8))=\mathbf{6 3}$ of $S U(8)$ vanishes, yielding [9]

$$
\left.\frac{\partial^{2} \mathcal{I}_{4, \mathcal{N}=8}}{\partial Z_{A B} \bar{\partial}^{B C}}\right|_{\mathbf{A d j}(S U(8))}=0 \Longleftrightarrow Z_{A C} \bar{Z}^{B C}=\frac{1}{2^{3}} \delta_{A}^{B} Z_{D E} Z^{D E}
$$

This is a mixed rank-2 $S U(8)$-covariant condition. By further differentiating with respect to the scalars $\phi$ parametrizing $\frac{E_{7(7)}}{S U(8)}$ and using the Maurer-Cartan Eqs. (3.3), one obtains another $S U$ (8)-covariant relation (notice the strict similarity to the $\mathcal{N}=8$, $d=4$ Attractor Eqs. (3.25) [9]:

$$
Z_{[A B} Z_{C D]}-\frac{1}{4 !} \epsilon_{A B C D E F G H} \bar{Z}^{E F} \bar{Z}^{G H}=0 .
$$

Actually, Eq. (3.40) form with Eq. 3.39 an $E_{7(7)}$-invariant set of differential conditions defining $\mathcal{O}_{\frac{1}{2}-B P S}$. Indeed, as noticed in [9], Eq. 3.40 can be rewritten as

$$
\frac{\partial^{2} \mathcal{I}_{4, \mathcal{N}=8}}{\partial Z_{[A B} \partial Z_{C D]}}-\frac{1}{4 !} \epsilon^{A B C D E F G H} \frac{\partial^{2} \mathcal{I}_{4, \mathcal{N}=8}}{\overline{\partial Z}^{[E F} \overline{\partial Z}^{G H]}}=0 .
$$

Thus, by using the notation $Z_{\mathbf{5 6}} \equiv\left(\mathcal{Z}, \mathcal{Z}^{T}\right)=\left(Z_{A B}, \bar{Z}^{A B}\right)$ (recall Eqs. 2.22 and $(2.23)$ ), Eqs. 3.39 and $(3.40)-(3.41)$ can be rewritten in the manifestly $E_{7(7)}$-invariant fashion

$$
\left.\frac{\partial^{2} \mathcal{I}_{4, \mathcal{N}=8}}{\partial Z_{\mathbf{5 6}} \partial Z_{\mathbf{5 6}}}\right|_{\mathbf{A d j}\left(E_{7(7)}\right)}=0
$$

where $\operatorname{Adj}\left(E_{7(7)}\right)=\mathbf{1 3 3}$ is the adjoint representation of $E_{7(7)}$. Notice that $\frac{\partial^{2} \mathcal{I}_{4, \mathcal{N}=8}}{\partial Z_{56} \partial Z_{\mathbf{5 6}}}$ is a rank-2 symmetric true-tensor $E_{7(7)}$-tensor, thus sitting in the symmetric product representation $(56 \times 56)_{s}=1596$ of $E_{7(7)}$, which in turns enjoys the following branching with respect to $E_{7(7)}[31,9]$ :

$$
(56 \times 56)_{s}=1596 \longrightarrow 1463+\underset{\operatorname{Adj}\left(E_{7(7)}\right)}{133} .
$$

It is here worth remarking that the constraints 3.39$)$ and $(3.40)-(3.41)$ (or equivalently $((3.42))$ ) imply the constraint (3.34), because in fact they are stronger constraints.

Along the $\frac{1}{2}$-BPS orbit it holds that

$$
\lambda_{1}=\lambda_{2}=\lambda_{3}=\lambda_{4}
$$

Furthermore, it can be shown that $\varphi_{Z}=2 k \pi, k \in \mathbb{Z}$, so the skew-eigenvalues of $Z_{A B}$ (see Eq. (3.10) ) are real. In agreement with the results of [4] and [5], the (maximal compact) symmetry of the skew-diagonalized central charge matrix $Z_{A B, s k e w-d i a g}$. all along the $\frac{1}{2}$-BPS small flow is $U S p(8)$, which is the mcs of the non-translational part of the stabilizer of $\mathcal{O}_{\frac{1}{2}-B P S}$, expressing the maximal compact symmetry of $\mathcal{O}_{\frac{1}{2}-B P S}$ itself. The counting of the 
parameters of $\mathcal{O}_{\frac{1}{2}-B P S}$ consistently reads: $28=1$ skew-eigenvalue $\lambda_{1}+27\left(=\operatorname{dim}_{\mathbb{R}}\left(\frac{\operatorname{SU}(8)}{\operatorname{USp}(8)}\right)\right)$ "generalized angles".

Interestingly, $U S p(8)$ also is the enhanced compact symmetry of $Z_{A B \text {,skew-diag. at the }}$ event horizon of the large non-BPS $Z_{A B} \neq 0$ attractor scalar flow (see Eq. (3.21) above). Indeed, the charge orbits $\mathcal{O}_{\text {non-BPS, }} Z_{A B} \neq 0$ and $\mathcal{O}_{\frac{1}{2}-B P S}$ (respectively given by Eqs. (3.18) and $(3.38)$ ) coincide, up to the translational factor $T_{27}$ in the stabilizer, and thus they have the same maximal compact symmetry.

As given by the analysis of [3], the classification of large and small orbits of the $\mathbf{5 6}$ of $E_{7(7)}$ can be performed also considering the symplectic basis composed by the fluxes $q_{\Lambda}(\Lambda=$ $1, \ldots, 56)$. In general, the symplectic basis of charges is useful in order to determine, through constraints imposed on the relevant $U$-invariant, the number and typology of orbits of the relevant representation of the $U$-duality group. On the other hand, using the manifestly $H$ covariant basis of central charges and matter charges one can achieve a symplectic-invariant characterization of charge orbits, and also study the related supersymmetry-preserving features.

Finally, it is worth pointing out once again that there is a crucial difference among the various constraints defining the two large and the three small charge orbits of $\mathcal{N}=8, d=4$ supergravity listed above:

- The large charge orbits $\mathcal{O}_{\frac{1}{8}-B P S \text {,large }}$ and $\mathcal{O}_{\text {non-BPS,Z }} \neq \begin{aligned} & \text { AB } \\ & \text {, }\end{aligned}$ (3.14) and (3.18), are in order defined by the $E_{7(7)}$-invariant conditions $\mathcal{I}_{4, \mathcal{N}=8}>0$ and $\mathcal{I}_{4, \mathcal{N}=8}<0$. Due to their $E_{7(7)}$-invariance, these conditions are identities for the scalar fields $\phi$ spanning $\frac{E_{7(7)}}{S U(8)}$. However, the classical attractor mechanism does hold for large extremal $\mathrm{BHs}$, and the scalars $\phi$ are stabilized purely in terms of charges $\mathcal{P}$ at the event horizon $\left(r \rightarrow r_{H}^{+}\right)$through the only two independent solutions (3.16) and (3.20) to the $\mathcal{N}=8, d=4$ Attractor Eqs. (3.24)-(3.25).

- The small charge orbits $\mathcal{O}_{\frac{1}{8}-B P S, \text { small }}, \mathcal{O}_{\frac{1}{4}-B P S}$ and $\mathcal{O}_{\frac{1}{2}-B P S}$, respectively given by Eqs. (3.31), (3.33) and (3.38), are in order defined by the $E_{7(7)}$-invariant conditions (3.30), (3.34) and (3.42). Due to their $E_{7(7)}$-invariance, these conditions are identities for the scalars $\phi$, which thus are not stabilized along such orbits. Indeed, the classical attractor mechanism does not hold for small BHs.

\section{$4 \mathcal{N}=4$}

In $\mathcal{N}=4, d=4$ supergravity, unlike the $\mathcal{N}=8$ case, matter (vector) multiplets appear (see e.g. [35, 36]). By denoting their number with $M$, the related scalar manifold is the symmetric coset

$$
\left(\frac{G}{H}\right)_{\mathcal{N}=4, d=4}=\frac{S L(2, \mathbb{R})}{U(1)} \times \frac{S O(6, M)}{S O(6) \times S O(M)}, \operatorname{dim}_{\mathbb{R}}=6 M+2 .
$$


The Abelian vector field strengths and their duals, as well the corresponding fluxes (charges), sit in the bi-fundamental $(\mathbf{2}, \mathbf{6}+\mathbf{M})$ representation of the global, classical (see Footnote 1) $U$-duality group $S L(2, \mathbb{R}) \times S O(6, M)$ [37. Such a representation determines the embedding of $S L(2, \mathbb{R}) \times S O(6, M)$ into the symplectic group $S p(12+2 M, \mathbb{R})$. The representation $(\mathbf{2}, \mathbf{6}+\mathbf{M})$ is endowed with a natural symplectic metric

$$
\Omega \equiv \epsilon_{\alpha \beta} \eta_{\Lambda \Sigma}
$$

where $\epsilon_{\alpha \beta}(\alpha, \beta=1,2)$ is the (inverse of the) $S L(2, \mathbb{R})$ skew-symmetric metric defined in Eq. (3.11), and $\eta_{\Lambda \Sigma}(\Lambda, \Sigma=1, \ldots, 6+M=n$; recall Eq. (2.26)) is the Lorentzian metric of $S O(6, M)$. Moreover, the $\mathcal{R}$-symmetry group is $U(4)$.

Furthermore, $(\mathbf{2}, \mathbf{6}+\mathbf{M})$ admits an unique invariant, which will be denoted by $\mathcal{I}_{4, \mathcal{N}=4}$ throughout. $\mathcal{I}_{4, \mathcal{N}=4}$ is quartic in charges, and it was firstly determined in [14, 19, 38].

More precisely, $\mathcal{I}_{4, \mathcal{N}=4}$ is the unique combination of "dressed" charges $Z_{A B}=Z_{[A B]}(\phi, \mathcal{P})$ (central charge matrix, $A, B=1, \ldots, 4)$ and $Z_{I}(\phi, \mathcal{P})$ (matter charges, $\left.I=1, \ldots, M\right)$ satisfying

$$
\partial_{\phi} \mathcal{I}_{4, \mathcal{N}=4}\left(Z_{A B}(\phi, \mathcal{P}), Z_{I}(\phi, \mathcal{P})\right)=0, \quad \forall \phi \in\left(\frac{G}{H}\right)_{\mathcal{N}=4, d=4} .
$$

Eq. 4.3 can be computed by using the Maurer-Cartan Eqs. of the coset $\frac{S L(2, \mathbb{R})}{U(1)} \times \frac{S O(6, M)}{S O(6) \times S O(M)}$ (see e.g. [29], and Refs. therein):

$$
\begin{aligned}
\nabla Z_{A B} & =\frac{1}{2} P \epsilon_{A B C D} \bar{Z}^{C D}+P_{A B I} \bar{Z}^{I} \\
\nabla Z_{I} & =\frac{1}{2} P_{A B I} \bar{Z}^{A B}+P \eta_{I J} \bar{Z}^{J}
\end{aligned}
$$

or equivalently by performing an infinitesimal $\frac{S L(2, \mathbb{R})}{U(1)} \times \frac{S O(6, M)}{S O(6) \times S O(M)}$-transformation of the central charge matrix and of matter charges (see e.g. [29], and Refs. therein):

$$
\begin{aligned}
\delta_{\left(\xi, \xi_{A B \mid I}\right)} Z_{A B} & =\frac{1}{2} \xi \epsilon_{A B C D} \bar{Z}^{C D}+\xi_{A B \mid I} Z^{I} \\
\delta_{\left(\xi, \xi_{A B \mid I}\right)} Z_{I} & =\bar{\xi} \eta_{I J} \bar{Z}^{J}+\frac{1}{2} \xi_{A B \mid I} \bar{Z}^{A B},
\end{aligned}
$$

where $\nabla$ stands for the covariant differential operator in $\frac{S L(2, \mathbb{R})}{U(1)} \times \frac{S O(6, M)}{S O(6) \times S O(M)} . P$ and $P_{A B I}$ respectively are the Vielbein 1-forms of $\frac{S L(2, \mathbb{R})}{U(1)}$ and $\frac{S O(6, M)}{S O(6) \times S O(M)}$, with $P_{A B I}$ satisfying the reality condition:

$$
P_{A B I}=\frac{1}{2} \eta_{I J} \epsilon_{A B C D} \bar{P}^{C D J}
$$

Moreover, $\xi$ is the infinitesimal $\frac{S L(2, \mathbb{R})}{U(1)}$-parameter and $\xi_{A B \mid I}$ are the infinitesimal $\frac{S O(6, M)}{S O(6) \times S O(M)}$ parameters, satisfying the reality condition

$$
\bar{\xi}^{A B \mid I}=\frac{1}{2} \eta^{I J} \epsilon^{A B C D} \xi_{C D \mid J}
$$


As found in [14, 19, 38] and rigorously re-obtained in [29], in terms of $Z_{A B}$ and $Z_{I}$ the unique solution of Eq. 4.3 reads:

$$
\mathcal{I}_{4, \mathcal{N}=4}=\mathcal{S}_{1}^{2}-\left|\mathcal{S}_{2}\right|^{2}
$$

where one can identify $\mathcal{S}_{1} \equiv L_{0}, \mathcal{S}_{2}=L_{1}+i L_{2}$, with $L \equiv\left(L_{0}, L_{1}, L_{2}\right)$ being an $S L(2, \mathbb{R}) \sim$ $S O(1,2)$-vector with square norm

$$
L^{2}=L_{0}^{2}-L_{1}^{2}-L_{2}^{2}=\mathcal{S}_{1}^{2}-\left|\mathcal{S}_{2}\right|^{2} .
$$

$\mathcal{S}_{1}$ and $\mathcal{S}_{2}$ are defined as [29]

$$
\begin{aligned}
\mathcal{S}_{1} & \equiv \frac{1}{2} Z_{A B} \bar{Z}^{A B}-Z_{I} \bar{Z}^{I} \in \mathbb{R} ; \\
\mathcal{S}_{2} & \equiv \frac{1}{4} \epsilon^{A B C D} Z_{A B} Z_{C D}-\bar{Z}_{I} \bar{Z}_{I} \in \mathbb{C} .
\end{aligned}
$$

In [29] it was indeed shown that $\mathcal{I}_{4, \mathcal{N}=4}$ given by Eq. 4.10 is the unique combination of $S O(6, M)$-invariant and scalar-dependent quantities, which is actually also $S L(2, \mathbb{R})$ independent and thus scalar-independent, satisfying

$$
\begin{aligned}
\delta_{\xi} \mathcal{I}_{4, \mathcal{N}}=4 & =0 ; \\
\delta_{\xi_{A B \mid I}} \mathcal{I}_{4, \mathcal{N}=4} & =0,
\end{aligned}
$$

with Eqs. (4.6), (4.7) and (4.9) holding true.

On the other hand, the expression of $\mathcal{I}_{4, \mathcal{N}=4}$ in terms of the "bare" charges $\mathcal{P}$ reads [14, 15, 18, 19]

$$
\mathcal{I}_{4, \mathcal{N}=4}=p^{2} q^{2}-(p \cdot q)^{2}=\frac{1}{2}\left(p_{\Lambda} q_{\Sigma}-p_{\Sigma} q_{\Lambda}\right)\left(p_{\Xi} q_{\Omega}-p_{\Omega} q_{\Xi}\right) \eta^{\Lambda \Xi} \eta^{\Sigma \Omega}=\frac{1}{2} T_{\Lambda \Sigma}^{(a)} T^{(a) \mid \Lambda \Sigma},
$$

where

$$
p^{2} \equiv p \cdot p \equiv p_{\Lambda} p_{\Sigma} \eta^{\Lambda \Sigma}, q^{2} \equiv q \cdot q \equiv q_{\Lambda} q_{\Sigma} \eta^{\Lambda \Sigma}, p \cdot q \equiv p_{\Lambda} q_{\Sigma} \eta^{\Lambda \Sigma},
$$

and the tensor

$$
T_{\Lambda \Sigma}^{(a)} \equiv p_{\Lambda} q_{\Sigma}-p_{\Sigma} q_{\Lambda}=T_{[\Lambda \Sigma]}^{(a)}
$$

has been introduced (the upperscript "(a)" stands for "anti-symmetric").

The classification of charge orbits, in particular the BPS ones, was performed in [3] and [9]. By performing a suitable $U(1) \times S O(6)(\sim U(4))$-transformation, the central charge matrix $Z_{A B}$ can be skew-diagonalized in the normal frame (recall definition (3.11)):

$$
Z_{A B} \stackrel{U(4)}{\longrightarrow} Z_{A B, \text { skew-diag. }} \equiv\left(\begin{array}{ll}
z_{1} & \\
& z_{2}
\end{array}\right) \otimes \epsilon, \quad z_{1}, z_{2} \in \mathbb{R}^{+},
$$

where the ordering $z_{1} \geqslant z_{2}$ does not imply any loss of generality. Furthermore, by performing a suitable $S O(M)$-transformation, the vector $Z_{I}$ of matter charges can be reduced to have only two non-vanishing entries, one real positive and the other one complex, say (without loss of generality, with the subscript "red." standing for "reduced")

$$
Z_{I} \stackrel{S O(M)}{\longrightarrow} Z_{I, \text { red. }} \equiv\left(\rho_{1} e^{i \theta}, \rho_{2}, 0, \ldots, 0\right), \quad \rho_{1}, \rho_{2} \in \mathbb{R}^{+}, \quad \theta \in \mathbb{R} .
$$


For non-vanishing (in general different) skew-eigenvalues $z_{1}$ and $z_{2}$, the symmetry group of

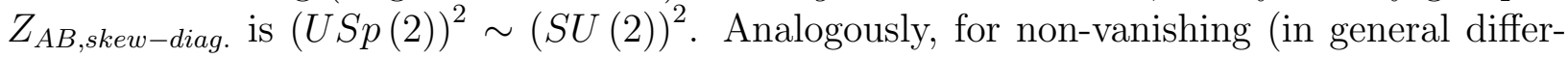
ent) $\rho_{1}$ and $\rho_{2}$ (and non-vanishing phase $\theta$ ) the symmetry group of $Z_{I, \text { red. }}$ is $S O(M-2)$. Thus, beside $z_{1}, z_{2}, \rho_{1}, \rho_{2}$ and $\theta$ the generic $Z_{A B}$ and $Z_{I}$ are described by $7+2 M=$ $\operatorname{dim}_{\mathbb{R}}\left(\frac{U(4) \times S O(M)}{(S U(2))^{2} \times S O(M-2)}\right)$ "generalized angles". Consistently, the total number of parameters is $2+2+1+7+2 M=12+2 M$, which is the real dimension of the bi-fundamental representation $(\mathbf{2}, \mathbf{6}+\mathbf{M})$, defining the embedding of $S L(2, \mathbb{R}) \times S O(6, M)$ into $S p(12+2 M, \mathbb{R})$.

In $\mathcal{N}=4, d=4$ matter coupled supergravity three distinct large charge orbits of the $(\mathbf{2}, \mathbf{6}+\mathbf{M})$ of $S L(2, \mathbb{R}) \times S O(6, M)$ (for which $\mathcal{I}_{4, \mathcal{N}=4} \neq 0$, and the attractor mechanism holds) exist, as resulting from the analysis performed in 7 [13]:

1. The large $\frac{1}{4}$-BPS orbit

$$
\mathcal{O}_{\frac{1}{4}-B P S, \text { large }}=S L(2, \mathbb{R}) \times \frac{S O(6, M)}{S O(4, M) \times S O(2)}, \operatorname{dim}_{\mathbb{R}}=11+2 M,
$$

is defined by the $S L(2, \mathbb{R}) \times S O(6, M)$-invariant constraint

$$
\mathcal{I}_{4, \mathcal{N}=4}>0 \text {. }
$$

Thus, the corresponding horizon solution of the $\mathcal{N}=4, d=4$ Attractor Eqs. yields [3, 9, 13 ]

$$
\begin{aligned}
& z_{1} \in \mathbb{R}_{0}^{+}, \quad z_{2}=0, \quad \rho_{1}=\rho_{2}=0, \quad \theta \text { undetermined; } \\
& \mathcal{S}_{1}=z_{1}^{2}>0, \mathcal{S}_{2}=0 .
\end{aligned}
$$

Therefore, at the event horizon, the symmetry group of $Z_{A B, s k e w-d i a g}$. defined in Eq. 4.19 does not get enhanced, while the symmetry group of $Z_{i, \text { red. }}$ defined in Eq. 4.20) gets enhanced as follows:

$$
S O(M-2) \stackrel{r \rightarrow r_{H}^{+}}{\longrightarrow} S O(M) .
$$

As a consequence, the horizon attractor solution exploits the maximal compact symmetry $S U(2) \times S U(2) \times S O(M) \times S O(2)$, which is the $m c s$ [31] of the stabilizer of $\mathcal{O}_{\frac{1}{4}-B P S \text {, large }}$ itself.

2. The large non-BPS $Z_{A B}=0$ orbit (existing for $M \geqslant 2$ ) [13]

$$
\mathcal{O}_{\text {non-BPS, } Z_{A B}=0, \text { large }}=S L(2, \mathbb{R}) \times \frac{S O(6, M)}{S O(6, M-2) \times S O(2)}, \operatorname{dim}_{\mathbb{R}}=11+2 M,
$$

is defined by the $S L(2, \mathbb{R}) \times S O(6, M)$-invariant constraint

$$
\mathcal{I}_{4, \mathcal{N}=4}>0 .
$$

\footnotetext{
${ }^{7}$ Consistent with the analysis of [13, Eqs. 4.21, 4.26 and 4.31 fix a slightly misleading notation for the large charge orbits of $\mathcal{N}=4, d=4$ matter coupled supergravity, as given by Table 1 of [39].
} 
Thus, the corresponding attractor solution of the $\mathcal{N}=4, d=4$ Attractor Eqs. yields (for $M \geqslant 2$ ) [3, 9, 13]

$$
\begin{aligned}
& z_{1}=z_{2}=0, \quad \rho_{1}^{2} e^{2 i \theta}+\rho_{2}^{2}=0 \Leftrightarrow \rho_{1}=\rho_{2} \in \mathbb{R}_{0}^{+}, \theta=\frac{\pi}{2}+k \pi, k \in \mathbb{Z} ; \\
& \mathcal{S}_{1}=-2 \rho_{1}^{2}<0, \mathcal{S}_{2}=0 .
\end{aligned}
$$

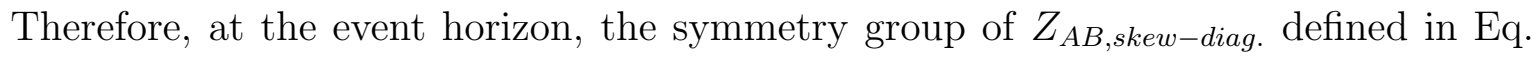
4.19) gets enhanced as follows:

$$
(S U(2))^{2} \stackrel{r \rightarrow r_{H}^{+}}{\longrightarrow} S U(4)
$$

and the symmetry group of $Z_{i, r e d}$. defined in Eq. 4.20 does not get enhanced. Consequently, the horizon attractor solution exploits the maximal compact symmetry $S U(4) \times S O(M-2) \times S O(2)$, which is the $m c s$ [31] of the stabilizer of $\mathcal{O}_{\text {non }-B P S, Z_{A B}=0, \text { large }}$ itself.

3. The large non-BPS $Z_{A B} \neq 0$ orbit (existing for $M \geqslant 1$ ) [13]

$$
\mathcal{O}_{\text {non }-B P S, Z_{A B} \neq 0, \text { large }}=S L(2, \mathbb{R}) \times \frac{S O(6, M)}{S O(5, M-1) \times S O(1,1)}, \operatorname{dim}_{\mathbb{R}}=11+2 M,
$$

is defined by the $S L(2, \mathbb{R}) \times S O(6, M)$-invariant constraint

$$
\mathcal{I}_{4, \mathcal{N}=4}<0 .
$$

At the event horizon of the extremal $\mathrm{BH}$, the solution of the $\mathcal{N}=4, d=4$ Attractor Eqs. yields (for $M \geqslant 1$ ) [3, 9, 13]

$$
\begin{aligned}
z_{1} & =z_{2}=\frac{\rho_{1}}{\sqrt{2}} \in \mathbb{R}_{0}^{+}, \quad \rho_{2}=0, \quad \theta=\frac{\pi}{2}+k \pi, k \in \mathbb{Z} \\
\mathcal{S}_{1} & =0, \mathcal{S}_{2}=3 z_{1}^{2}>0
\end{aligned}
$$

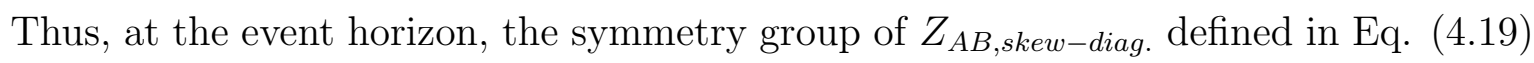
gets enhanced as follows:

$$
(S U(2))^{2} \stackrel{r \rightarrow r_{H}^{+}}{\longrightarrow} U S p(4),
$$

and the symmetry group of $Z_{i, r e d}$. defined in Eq. 4.20 gets also enhanced as

$$
S O(M-2) \stackrel{r \rightarrow r_{H}^{+}}{\longrightarrow} S O(M-1)
$$

As a consequence, the horizon attractor solution exploits the maximal compact symmetry $U S p(4) \times S O(M-1)$ which, due to the isomorphism $U S p(4) \sim S O(5)$, is the

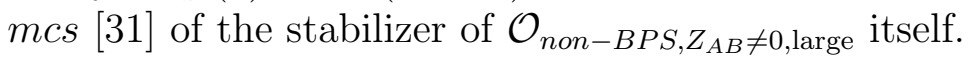


As mentioned above, for such large charge orbits, corresponding to a non-vanishing quartic $S L(2, \mathbb{R}) \times S O(6, M)$-invariant $\mathcal{I}_{4, \mathcal{N}=4}$ and thus supporting large BHs, the attractor mechanism holds. Consequently, the computations of the Bekenstein-Hawking BH entropy can be performed by solving the criticality conditions for the "BH potential"

$$
V_{B H, \mathcal{N}=4}=\frac{1}{2} Z_{A B} \bar{Z}^{A B}+Z_{I} \bar{Z}^{I}
$$

the result being

$$
\frac{S_{B H,}}{\pi}=\left.V_{B H, \mathcal{N}=4}\right|_{\partial V_{B H, \mathcal{N}=4}=0}=V_{B H, \mathcal{N}=4}\left(\phi_{H}(\mathcal{P}), \mathcal{P}\right)=\left|\mathcal{I}_{4, \mathcal{N}=4}\right|^{1 / 2},
$$

where $\phi_{H}(\mathcal{P})$ denotes the set of solutions to the criticality conditions of $V_{B H, \mathcal{N}=4}$, namely the Attractor Eqs. of $\mathcal{N}=4, d=4$ matter coupled supergravity:

$$
\partial_{\phi} V_{B H, \mathcal{N}=4}=0, \quad \forall \phi \in \frac{S L(2, \mathbb{R})}{U(1)} \times \frac{S O(6, M)}{S O(6) \times S O(M)},
$$

expressing the stabilization of the scalar fields purely in terms of supporting charges $\mathcal{P}$ at the event horizon of the extremal BH. Through Eqs. 4.4-(4.5) and (4.37), Eqs. 4.39) can be rewritten as follows [13]:

$$
\left\{\begin{array}{l}
\left(\bar{Z}^{A B}+\frac{1}{2} \epsilon^{A B C D} Z_{C D}\right) Z^{I}=0 \\
Z^{I} Z^{J} \delta_{I J}+\frac{1}{4} \epsilon_{A B C D} \bar{Z}^{A B} \bar{Z}^{C D}=0
\end{array}\right.
$$

Actually, the critical potential $\left.V_{B H, \mathcal{N}=4}\right|_{\partial V_{B H, \mathcal{N}=4}=0}$ exhibits some "flat" directions, so not all scalars are stabilized in terms of charges at the event horizon [39]. Thus, Eq. (4.38) yields that the unstabilized scalars, spanning a related moduli space of the considered class of attractor solutions, do not enter in the expression of the BH entropy at all. The moduli spaces exhibited by the Attractor Eqs. 4.39)-4.40) are [39]

$$
\begin{aligned}
\mathcal{M}_{\frac{1}{4}-B P S, \text { large }} & =\frac{S O(4, M)}{S U(2) \times S U(2) \times S O(M)}, \operatorname{dim}_{\mathbb{R}}=4 M \\
\mathcal{M}_{\text {non-BPS, } Z_{A B}=0, \text { large }} & =\frac{S O(6, M-2)}{S U(4) \times S O(M-2)}, \operatorname{dim}_{\mathbb{R}}=6(M-2) \\
\mathcal{M}_{\text {non-BPS, } Z_{A B} \neq 0, \text { large }} & =S O(1,1) \times \frac{S O(5, M-1)}{U S p(4) \times S O(M-1)}, \operatorname{dim}_{\mathbb{R}}=5(M-1)+1 .
\end{aligned}
$$

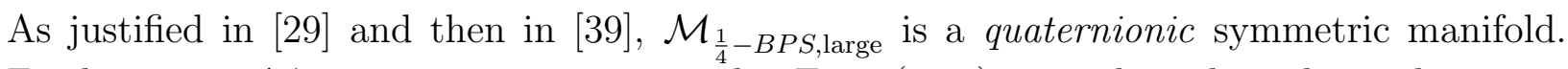

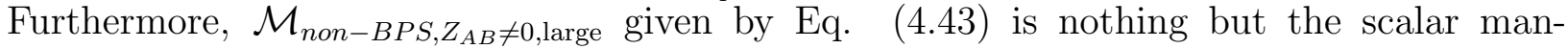
ifold of $\mathcal{N}=4, d=5$ matter coupled supergravity. The stabilizers of $\mathcal{M}_{\frac{1}{4}-B P S \text {, large }}$, $\mathcal{M}_{\text {non-BPS, }} Z_{A B}=0$, large and $\mathcal{M}_{n o n-B P S, Z_{A B} \neq 0 \text {, large }}$ exploit the maximal compact symmetry of the corresponding charge orbits; this symmetry becomes fully manifest through the enhancement of the compact symmetry group of $Z_{A B, \text { skew-diag. and }} Z_{I, \text { red. }}$ at the event horizon of the extremal BH, respectively given by Eqs. (4.25), 4.30) and (4.35)-4.36). 
Let us now analyze the small charge orbits of the $(\mathbf{2}, \mathbf{6}+\mathbf{M})$ of $S L(2, \mathbb{R}) \times S O(6, M)$, associated to $\mathcal{I}_{4, \mathcal{N}=4}=0$, for which the attractor mechanism does not hold. The analysis performed below completes the one given in [3] and [9].

While in $\mathcal{N}=8, d=4$ supergravity all three small charge orbits are BPS (with various degrees of supersymmetry-preservation), in the considered $\mathcal{N}=4, d=4$ theory there are five small charge orbits, two of them being $\frac{1}{2}$-BPS, one $\frac{1}{4}$-BPS, and the other two non-BPS (one with $Z_{A B}=0$ and the other with $Z_{A B} \neq 0$ ). Such an abundance of different charge orbits can be traced back to the factorized nature of the $U$-duality group $S L(2, \mathbb{R}) \times S O(6, M)$. Furthermore, it should be remarked that in $\mathcal{N}=4, d=4$ supergravity the $\frac{1}{(\mathcal{N}=) 4}$-BPS charge orbit exists only in its large version, differently from the $d=4$ maximal theory, in which both large and small $\frac{1}{(\mathcal{N}=) 8}$-BPS charge orbits exist.

It is now convenient to denote with $\alpha_{1}$ and $\alpha_{2}$ the two real non-negative eigenvalues of the matrix $Z_{A B} \bar{Z}^{C B}=\left(Z Z^{\dagger}\right)_{A}^{C}$. By recalling Eq. 4.19$)$, one can notice that $(i=1,2)$

$$
\alpha_{i}=z_{i}^{2}
$$

and one can order them as $\alpha_{1} \geqslant \alpha_{2}$, without any loss of generality. The explicit expression of $\alpha_{i}$ in terms of $U(4) \times S O(M)$-invariants (namely of $\operatorname{Tr}\left(Z Z^{\dagger}\right), \operatorname{Tr}\left(\left(Z Z^{\dagger}\right)^{2}\right)$, and suitable powers) is given by Eqs. (5.108) and (5.109) of [9].

Firstly, let us observe that from Eqs. (4.16) and (4.11) the $S L(2, \mathbb{R}) \times S O(6, M)$-invariant "degeneracy" condition can be written in the "dressed" ( $\mathcal{R}$-symmetry- and $S O(M)$ - covariant) and "bare" (symplectic-, i.e. $S p(12+2 M, \mathbb{R})$ - covariant) charges' bases respectively as follows:

$$
\mathcal{I}_{4, \mathcal{N}=4}=0 \Leftrightarrow \mathcal{S}_{1}^{2}=\left|\mathcal{S}_{2}\right|^{2} \Leftrightarrow p^{2} q^{2}=(p \cdot q)^{2} \geqslant 0 .
$$

Then, in order to determine the number and typology of small orbits, it is convenient to start differentiating $\mathcal{I}_{4, \mathcal{N}=4}$ in the symplectic "bare" charges' basis $\mathcal{P} \equiv\left(p^{\Lambda}, q_{\Lambda}\right)^{T}$ (recall definition (1.2)). Eqs. (4.16) and (4.18) yield the constraints defining the "small" critical orbits to read

$$
\begin{aligned}
& \frac{\partial \mathcal{I}_{4, \mathcal{N}=4}}{\partial p_{\Lambda}}=2\left[q^{2} p^{\Lambda}-(q \cdot p) q^{\Lambda}\right]=2 T^{(a) \mid \Lambda \Sigma} q_{\Sigma}=0 \\
& \frac{\partial \mathcal{I}_{4, \mathcal{N}=4}}{\partial q_{\Lambda}}=2\left[p^{2} q^{\Lambda}-(q \cdot p) p^{\Lambda}\right]=-2 T^{(a) \mid \Lambda \Sigma} p_{\Sigma}=0 .
\end{aligned}
$$

Due to the definition (4.18), or equivalently to the homogeneity (of degree four) in charges of $\mathcal{I}_{4, \mathcal{N}=4}$, it is worth noticing that the "criticality" constraints (4.46) and (4.47) imply the "degeneracy" condition 4.45).

Beside the trivial one $\left(p_{\Lambda}=0=q_{\Lambda} \forall \Lambda\right)$, all the solutions to the "criticality" constraints 
4.46) and 4.47 list as follows:

$$
\begin{aligned}
& A]\left\{\begin{array}{l}
T_{\Lambda \Sigma}^{(a)}=0 ; \\
\left\{\begin{array}{l}
p^{2} q^{2}=(p \cdot q)^{2}>0:\left\{\begin{array}{l}
A .1] p^{2}>0, q^{2}>0 ; \\
\text { aut } \\
\text { A.2] } p^{2}<0, q^{2}<0 ;
\end{array}\right. \\
\text { A.3] } p^{2} q^{2}=(p \cdot q)^{2}=0: p^{2}=0, q^{2}=0 ;
\end{array}\right.
\end{array}\right. \\
& B]\left\{\begin{array}{l}
T_{\Lambda \Sigma}^{(a)} \neq 0 \\
p^{2}=q^{2}=p \cdot q=0 .
\end{array}\right.
\end{aligned}
$$

Notice that each set $(\mathbf{A . 1}, \mathbf{A . 2}, \mathbf{A . 3}$ and $\mathbf{B})$ of constraints is $S L(2, \mathbb{R}) \times S O(6, M)$-invariant, but formulated in terms of the symplectic charge basis $\mathcal{P}$.

The solutions $4.48-4.49$ can be rewritten by noticing that $\frac{\partial^{2} \mathcal{I}_{4, \mathcal{N}=4}}{\partial \mathcal{P} \partial \mathcal{P}}$, i.e. the tensor of second derivatives of $\mathcal{I}_{4, \mathcal{N}=4}$ with respect to $\mathcal{P}$, sits in the symmetric product representation $((\mathbf{2}, \mathbf{6}+\mathbf{M}) \times(\mathbf{2}, \mathbf{6}+\mathbf{M}))_{s}$ of the $U$-duality group $S L(2, \mathbb{R}) \times S O(6, M)$, which decomposes as follows [9]:

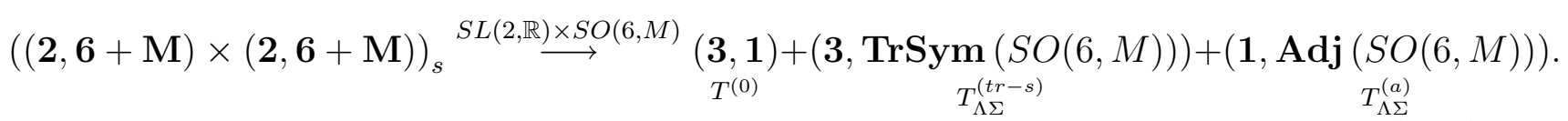

The antisymmetric tensor

$$
\left.T_{\Lambda \Sigma}^{(a)} \equiv \frac{\partial^{2} \mathcal{I}_{4, \mathcal{N}=4}}{\partial \mathcal{P} \partial \mathcal{P}}\right|_{(\mathbf{1}, \mathbf{A d j}(S O(6, M)))}
$$

was already introduced in Eq. 4.18. TrSym and Adj respectively denote the traceless symmetric and adjoint representations, and [9]

$$
\begin{aligned}
T_{\Lambda \Sigma}^{(t r-s)} & \left.\equiv \frac{\partial^{2} \mathcal{I}_{4, \mathcal{N}=4}}{\partial \mathcal{P} \partial \mathcal{P}}\right|_{(\mathbf{3}, \operatorname{TrSym}(S O(6, M)))} \equiv \\
& \equiv\left(q_{\Lambda} q_{\Sigma}-\frac{q^{2}}{6+M} \eta_{\Lambda \Sigma}, p_{\Lambda} p_{\Sigma}-\frac{p^{2}}{6+M} \eta_{\Lambda \Sigma}, \frac{1}{2}\left(q_{\Lambda} p_{\Sigma}+q_{\Sigma} p_{\Lambda}\right)-\frac{q \cdot p}{6+M} \eta_{\Lambda \Sigma}\right) ; \\
T^{(0)} & \left.\equiv \frac{\partial^{2} \mathcal{I}_{4, \mathcal{N}=4}}{\partial \mathcal{P} \partial \mathcal{P}}\right|_{(\mathbf{3}, \mathbf{1})} \equiv \operatorname{Tr}_{S O(6, M)}\left(T_{\Lambda \Sigma}^{(s)}\right) \equiv \operatorname{Tr}_{S O(6, M)}\left(\left.\frac{\partial^{2} \mathcal{I}_{4, \mathcal{N}=4}}{\partial \mathcal{P} \partial \mathcal{P}}\right|_{(\mathbf{3 , S y m}(S O(6, M)))}\right)= \\
& =\left(q^{2}, p^{2}, q \cdot p\right)=\left(\begin{array}{cc}
q^{2} & q \cdot p \\
q \cdot p & p^{2}
\end{array}\right) .
\end{aligned}
$$


The definition 4.53) of $T^{(0)}$ implies that (recall Eq. 4.16)

$$
\mathcal{I}_{4, \mathcal{N}=4}=\operatorname{det}\left(T^{(0)}\right)=\operatorname{det}\left(\left.\frac{\partial^{2} \mathcal{I}_{4, \mathcal{N}=4}}{\partial \mathcal{P} \partial \mathcal{P}}\right|_{(\mathbf{3}, \mathbf{1})}\right),
$$

in turn yielding another, equivalent $S L(2, \mathbb{R}) \times S O(6, M)$-invariant characterization of the "degeneracy" condition 4.45):

$$
\operatorname{det}\left(T^{(0)}\right)=\operatorname{det}\left(\left.\frac{\partial^{2} \mathcal{I}_{4, \mathcal{N}=4}}{\partial \mathcal{P} \partial \mathcal{P}}\right|_{(\mathbf{3 , 1})}\right)=0
$$

Thus, Eqs. 4.48)-4.49) can be recast as follows:

$$
\begin{aligned}
& A]\left\{\begin{array}{l}
T_{\Lambda \Sigma}^{(a)}=0 ; \\
\operatorname{det}\left(T^{(0)}\right)=0,\left\{\begin{array}{l}
\text { A.1] } \operatorname{Tr}\left(T^{(0)}\right)>0 ; \\
\text { aut } \\
\text { A.2] } \operatorname{Tr}\left(T^{(0)}\right)<0 ; \\
\text { aut } \operatorname{Tr}\left(T^{(0)}\right)=0 \Leftrightarrow T^{(0)}=0 ; \\
\text { A.3] }
\end{array}\right.
\end{array}\right. \\
& B]\left\{\begin{array}{l}
T_{\Lambda \Sigma}^{(a)} \neq 0 ; \\
T^{(0)}=0 .
\end{array}\right.
\end{aligned}
$$

As mentioned above, each set (A.1, A.2, A.3 and B) of constraints is $S L(2, \mathbb{R}) \times S O(6, M)$ invariant, but formulated in terms of the symplectic charge basis $\mathcal{P}$.

It is interesting to point out that, differently from $\mathcal{N}=8, d=4$ supergravity treated in Sect. 3, in $\mathcal{N}=4, d=4$ supergravity there are no small doubly-critical (or with higher degree of criticality) charge orbits independent from the small critical ones. This can be easily seen by noticing that the solutions (4.56)-(4.57) to the "criticality" constraints (4.46)

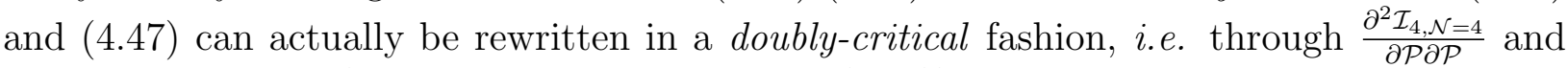
related projections (according to decomposition (4.50). For completeness' sake, we report here the second order derivatives of $\mathcal{I}_{4, \mathcal{N}=4}$ with respect to the "bare" symplectic charges:

$$
\begin{aligned}
& \frac{\partial^{2} \mathcal{I}_{4, \mathcal{N}=4}}{\partial p_{\Sigma} \partial p_{\Lambda}}=2\left(q^{2} \eta^{\Lambda \Sigma}-q^{\Lambda} q^{\Sigma}\right) ; \\
& \frac{\partial^{2} \mathcal{I}_{4, \mathcal{N}=4}}{\partial q_{\Sigma} \partial q_{\Lambda}}=2\left(p^{2} \eta^{\Lambda \Sigma}-p^{\Lambda} p^{\Sigma}\right) ; \\
& \frac{\partial^{2} \mathcal{I}_{4, \mathcal{N}=4}}{\partial q_{\Sigma} \partial p_{\Lambda}}=4 T^{(a) \mid \Lambda \Sigma} .
\end{aligned}
$$

In order to determine the small orbits of the bi-fundamental representation $(\mathbf{2}, \mathbf{6}+\mathbf{M})$ of the $U$-duality group $S L(2, \mathbb{R}) \times S O(6, M)$ and to study their supersymmetry-preserving 
properties, it is now convenient to switch to the basis of "dressed" charges (recall Eqs. 2.22) and (2.23)

$$
\mathcal{U} \equiv(\mathcal{Z}, \overline{\mathcal{Z}})^{T}=\left(Z_{A B}, Z^{I}, \bar{Z}_{A B}, \bar{Z}^{I}\right)^{T}
$$

From the analysis of [9], one obtains the following equivalence:

$$
\left.T_{\Lambda \Sigma}^{(a)} \equiv \frac{\partial^{2} \mathcal{I}_{4, \mathcal{N}=4}}{\partial \mathcal{P} \partial \mathcal{P}}\right|_{\operatorname{Adj}(S O(6, M))}=\left.0 \Leftrightarrow \frac{\partial^{2} \mathcal{I}_{4, \mathcal{N}=4}}{\partial \mathcal{U} \partial \mathcal{U}}\right|_{\operatorname{Adj}(S O(6, M))}=0 .
$$

The $S L(2, \mathbb{R}) \times S O(6, M)$-invariant constraint (4.62) is common to the small critical charge orbits determined by the solutions A.1, A.2 and A.3 of Eqs. (4.56). It also implies that $\alpha_{1}=\alpha_{2}\left[9\right.$. Then, the further $S L(2, \mathbb{R}) \times S O(6, M)$-invariant constraints $\operatorname{Tr}\left(T^{(0)}\right) \gtreqless 0$ can equivalently be rewritten as (recall definition 4.12 )

$$
\operatorname{Tr}\left(T^{(0)}\right) \gtreqless 0 \Leftrightarrow \mathcal{S}_{1} \gtreqless 0 .
$$

Therefore, one can characterize the small critical orbits A.1, A.2 and A.3 of Eqs. (4.48) and 4.56 as follows:

$$
A]\left\{\begin{array}{l}
\left.\frac{\partial^{2} \mathcal{I}_{4, \mathcal{N}=4}}{\partial \mathcal{U} \partial \mathcal{U}}\right|_{\operatorname{Adj}(S O(6, M))}=0 ; \\
\mathcal{S}_{1}^{2}=\left|\mathcal{S}_{2}\right|^{2},\left\{\begin{array}{l}
\text { A.1] } \mathcal{S}_{1}>0 ; \\
\text { aut } \\
\text { A.2] } \mathcal{S}_{1}<0 ; \\
\text { aut } \\
\text { A.3] } \mathcal{S}_{1}=0 \Leftrightarrow \mathcal{S}_{2}=0 .
\end{array}\right.
\end{array}\right.
$$

Notice that each set $(\mathbf{A . 1}, \mathbf{A . 2}, \mathbf{A . 3}$ and B $)$ of constraints is $S L(2, \mathbb{R}) \times S O(6, M)$-invariant but, differently from Eqs. 4.48) and 4.56), it is also independent from the symplectic basis eventually considered.

On the other hand, the $S L(2, \mathbb{R}) \times S O(6, M)$-invariant constraints (4.49) and (4.57) defining the small critical orbit $\mathbf{B}$ can be recast in a form which (differently from Eqs. 4.49) and (4.57) ) is independent from the symplectic basis eventually considered, as follows:

$$
B]\left\{\begin{array}{l}
\left.\frac{\partial^{2} \mathcal{I}_{4, \mathcal{N}=4}}{\partial \mathcal{U} \partial \mathcal{U}}\right|_{\operatorname{Adj}(S O(6, M))} \neq 0 ; \\
\mathcal{S}_{1}^{2}=\left|\mathcal{S}_{2}\right|^{2}=0 .
\end{array}\right.
$$

Thus, five distinct small charge orbits (all with $\mathcal{I}_{4, \mathcal{N}=4}=0$ ) exist:

1. The critical orbit A.1 is defined by the $S L(2, \mathbb{R}) \times S O(6, M)$-invariant constraints (4.48) (or 4.56), or 4.64). Such constraints are solved by the following flow solution (exhibiting maximal symmetry):

$$
z_{1}=z_{2} \in \mathbb{R}_{0}^{+}, \rho_{1}=\rho_{2}=0, \theta \text { undetermined }
$$


Thus, from the reasoning performed at the end of Sect. 2 and the analysis of [9], the considered small critical orbit is $\frac{1}{2}$-BPS. Along the corresponding small critical $\frac{1}{2}$-BPS flow, the (maximal compact) symmetry of the skew-diagonalized central charge matrix $Z_{A B, \text { skew-diag. }}$ defined in Eq. 44.19 is $U S p(4)$, whereas the one of $Z_{I, \text { red. }}$ defined in Eq. 4.20 is $S O(M)$. Therefore, the resulting maximal compact symmetry of the critical orbit A.1 is $U S p(4) \times S O(M)$.

2. The critical orbit $\mathbf{A . 2}$ is defined by the $S L(2, \mathbb{R}) \times S O(6, M)$-invariant constraints 4.48) (or 4.56), or 4.64). Such constraints are solved by the following flow solution, existing for $M \geqslant 1$ (and exhibiting maximal symmetry)

$$
z_{1}=z_{2}=0, \rho_{1} \in \mathbb{R}_{0}^{+}, \rho_{2}=0 .
$$

Thus, the considered small critical orbit is non-BPS $Z_{A B}=0$. Along the corresponding small critical non-BPS $Z_{A B}=0$ flow, the (maximal compact) symmetry of the

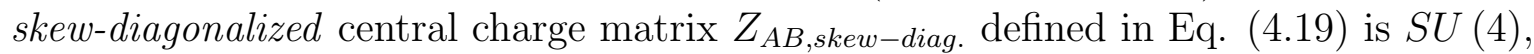
whereas the one of $Z_{I, \text { red }}$. defined in Eq. (4.20) is $S O(M-1)$. Therefore, the resulting maximal compact symmetry of the critical orbit A.2 is $S U(4) \times S O(M-1)$.

3. The critical orbit $\mathbf{A . 3}$ is defined by the $S L(2, \mathbb{R}) \times S O(6, M)$-invariant constraints (4.48) (or 4.56), or (4.64)). Such constraints are solved by the following flow solution, existing for $M \geqslant 1$ (and exhibiting maximal symmetry)

$$
z_{1}=z_{2}=\frac{\rho_{2}}{\sqrt{2}} \in \mathbb{R}_{0}^{+}, \rho_{1}=0, \theta \text { undetermined }
$$

This small critical orbit is $\frac{1}{2}$-BPS. Along the corresponding small critical non-BPS $Z_{A B} \neq 0$ flow, the (maximal compact) symmetry of the skew-diagonalized central

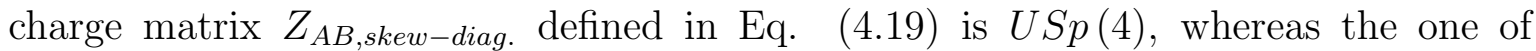
$Z_{I, \text { red. }}$. defined in Eq. (4.20) is $S O(M-1)$. Therefore, the resulting maximal compact symmetry of the critical orbit $\mathbf{A . 3}$ is $U S p(4) \times S O(M-1)$.

4. The critical orbit $\mathbf{B}$ is defined by the $S L(2, \mathbb{R}) \times S O(6, M)$-invariant constraints $(4.49)$ (or 4.57), or (4.65)). Such constraints are solved by the following flow solution, existing for $M \geqslant 2$ (and exhibiting maximal symmetry)

$$
\begin{aligned}
z_{1} & \in \mathbb{R}_{0}^{+}, z_{2}=0, \rho_{1}=\rho_{2}=\frac{z_{1}}{\sqrt{2}} ; \\
\theta & =\frac{\pi}{2}+k \pi, k \in \mathbb{Z} .
\end{aligned}
$$

This small critical orbit is $\frac{1}{4}$-BPS. Along the corresponding small critical non-BPS $Z_{A B} \neq 0$ flow, the (maximal compact) symmetry of the skew-diagonalized central charge matrix $Z_{A B, \text { skew-diag. }}$ defined in Eq. $\sqrt{4.19}$ is $(S U(2))^{2}$, whereas the one of $Z_{I, \text { red. }}$ defined in Eq. (4.20) is $S O(M-2)$. Therefore, the resulting maximal compact symmetry of the critical orbit $\mathbf{B}$ is $(S U(2))^{2} \times S O(M-2)$. 
5. The generic small lightlike case is defined by the $S L(2, \mathbb{R}) \times S O(6, M)$-invariant constraints 4.45) (or 4.55)). In this case, it is more convenient to consider the symplectic basis of "bare" charges $\mathcal{P}$ and, in order to determine the maximal compact symmetry of the flow solution(s), one can consider the saturation of the bound (4.45), namely:

$$
p^{2} q^{2}=(p \cdot q)^{2}=0
$$

This is in general solved by $p^{2}=0, p \cdot q=0$ and $q^{2} \neq 0$ (or equivalently by $q^{2}=0$, $p \cdot q=0$ and $\left.p^{2} \neq 0\right)$. It is easy to realize that the maximal compact symmetry of the flow solution is $S O(4) \times S O(M-1)$ in the case $q^{2}>0$, and $S O(5) \times S O(M-2)$ in the case $q^{2}<0$. In the first case the solution exists for $M \geqslant 1$, whereas in the second case the solution exists for $M \geqslant 2$. Thus, one actually gets two generic small lightlike orbits, both non-BPS $Z_{A B} \neq 0$, with maximal compact symmetry respectively given by $S O(4) \times S O(M-1)$ and $S O(5) \times S O(M-2)$.

Mutatis mutandis, the same considerations made at the end of Sect. 3 for $\mathcal{N}=8, d=4$ supergravity also hold for $\mathcal{N}=4, d=4$ matter coupled supergravity.

Notice that in pure $\mathcal{N}=4, d=4$ supergravity only the small $\frac{1}{2}$-BPS orbit A.1 and the large $\frac{1}{4}$-BPS orbit exist. Indeed, the non-BPS $Z_{A B} \neq 0$ and non-BPS $Z_{A B}=0$ large orbits and the small orbits A.2, A.3 and $\mathbf{B}$ cannot be realized, and the small lightlike orbit(s) of point 5 above coincide with small orbit A.1.

Finally, it is worth noticing that the $U(1)$ (stabilizer of the factor $\frac{S L(2, \mathbb{R})}{U(1)}$ of the scalar manifold (4.1) is broken both in large and small charge orbits, because both the central charge matrix $Z_{A B}$ and the matter charges $Z_{I}$ are charged with respect to it.

\section{$5 \mathcal{N}=2$}

In $\mathcal{N}=2, d=4$ supergravity one can repeat the analysis of [1, 40] (see also [41]), by using the properties of special Kähler geometry (SKG, see e.g. [22], and Refs. therein). Indeed, in SKG one can define an $S p(2 n, \mathbb{R})$ matrix over the scalar manifold (as in Eq. (2.9p), as well complex matrices $f$ and $h$ (as in Eqs. 2.10)-(2.14), without the need for the manifold to be necessarily a(n at least locally) symmetric space (see e.g. [21, 13]).

The basic identities of SKG applied to the (covariantly holomorphic) $\mathcal{N}=2, d=4$ central charge section

$$
Z \equiv e^{K / 2}\left(X^{\Lambda} q_{\Lambda}-F_{\Lambda} p^{\Lambda}\right)
$$

of the $U(1)$ Kähler-Hodge bundle (with Kähler weights $(1,-1))$ read as follows [20] $(i, \bar{j}=$ $1, \ldots, n-1$, with $n-1$ denoting the number of Abelian vector multiplets coupled to the supergravity one)

$$
\begin{aligned}
\bar{D}_{\bar{i}} Z & =0 \\
D_{i} D_{j} Z & =i C_{i j k} g^{k \bar{k}} \bar{D}_{\bar{k}} \bar{Z} \\
\bar{D}_{\bar{j}} D_{i} Z & =g_{i j} Z
\end{aligned}
$$


where $\left(X^{\Lambda}, F_{\Lambda}\right)$ are the holomorphic symplectic sections of the $U(1)$ Kähler-Hodge bundle (with Kähler weights $(2,0)$ ), and $K$ denotes the Kähler potential of the Abelian vector multiplets' scalar manifold, with metric $g_{i \bar{j}}=\bar{\partial} \bar{j} \partial_{i} K . \quad C_{i j k}$ is the rank-3 symmetric and covariantly holomorphic $C$-tensor of SKG (see e.g. [22], and Refs. therein):

$$
\begin{aligned}
\bar{D}_{\bar{l}} C_{i j k} & =0 ; \\
D_{[l} C_{i] j k} & =0 .
\end{aligned}
$$

Thus, in $\mathcal{N}=2, d=4$ supergravity coupled to $n-1$ Abelian vector multiplets, the " $B H$ potential" is given by [18, 19]

$$
V_{B H}(\phi, \mathcal{P})=Z \bar{Z}+g^{i \bar{j}}\left(D_{i} Z\right) \bar{D} \bar{j} \bar{Z}
$$

and the Attractor Eqs. read [20]

$$
\partial_{i} V_{B H}=0 \Leftrightarrow 2 \bar{Z} D_{i} Z+i C_{i j k} g^{j \bar{j}} g^{k \bar{k}}\left(\bar{D}_{\bar{j}} \bar{Z}\right) \bar{D}_{\bar{k}} \bar{Z}=0 .
$$

1. The ( $\frac{1}{2}$-BPS) supersymmetric solution to Attractor Eqs. 5.8 is determined by

$$
\left(D_{i} Z\right)_{\frac{1}{2}-B P S}=0, \forall i
$$

and therefore Eq. (5.7) yields

$$
V_{B H, \frac{1}{2}-B P S}=|Z|_{\frac{1}{2}-B P S}^{2},
$$

and the corresponding Hessian matrix of $V_{B H}$ has block components given by [20]

$$
\begin{aligned}
&\left(D_{i} \partial_{j} V_{B H}\right)_{\frac{1}{2}-B P S}=\left(\partial_{i} \partial_{j} V \partial_{B H}\right)_{\frac{1}{2}-B P S}=0 ; \\
&\left(\partial_{i} \bar{\partial}_{\bar{j}} V_{B H}\right)_{\frac{1}{2}-B P S}=2 g_{i j, \frac{1}{2}-B P S}|Z|_{\frac{1}{2}-B P S}^{2},
\end{aligned}
$$

showing that there are no "flat" directions for such the $\left(\frac{1}{2}-\right)$ BPS class of solutions to Attractor Eqs. (5.8) [33].

2. Non-supersymmetric (non-BPS) solutions to Attractor Eqs. (5.8) have $D_{i} Z \neq 0$ (at least) for some $i \in\{1, \ldots, n-1\}$. Generally, such solutions fall into two class [6], and they exhibit "flat" directions of $V_{B H}$ itself [33]. The non-BPS, $Z=0$ class is defined by the following constraints:

$$
D_{i} Z=\partial_{i} Z \neq 0 \text {, for some } i, Z=0 \text {, }
$$

thus yielding (from Eqs. (5.8)

$$
\left[C_{i j k} g^{j \bar{j}} g^{k \bar{k}}(\bar{\partial} \overline{\bar{j}} \bar{Z}) \bar{\partial}_{\bar{k}} \bar{Z}\right]_{n o n-B P S, Z=0}=0 .
$$

Thus, Eqs. (5.7) and (5.13) yield

$$
V_{B H, n o n-B P S, Z=0}=\left[g^{i \bar{j}}\left(D_{i} Z\right) \bar{D} \overline{\bar{j}} \bar{Z}\right]_{n o n-B P S, Z=0}=\left[g^{i \bar{j}}\left(\partial_{i} Z\right) \bar{\partial} \bar{j} \bar{Z}\right]_{n o n-B P S, Z=0} .
$$


3. The non-BPS, $Z \neq 0$ class is defined by the following constraints:

$$
D_{i} Z \neq 0 \text {, for some } i, Z \neq 0 \text {. }
$$

It is worth remarking that Eqs. (5.8) and the non-BPS $Z \neq 0$ defining constraints (5.16) imply the following relations to hold at the non-BPS $Z \neq 0$ critical points of $V_{B H}[13]:$

$$
\left[g^{i \bar{j}}\left(D_{i} Z\right) \bar{D} \overline{\bar{j}}\right]_{n o n-B P S, Z \neq 0}=-\frac{i}{2}\left[\frac{N_{3}(\bar{Z})}{\bar{Z}}\right]_{n o n-B P S, Z \neq 0}=\frac{i}{2}\left[\frac{\bar{N}_{3}(Z)}{Z}\right]_{n o n-B P S, Z \neq 0},
$$

where the cubic form $N_{3}(\bar{Z})$ is defined as 13

$$
N_{3}(\bar{Z}) \equiv C_{i j k} \bar{Z}^{i} \bar{Z}^{j} \bar{Z}^{k} \Leftrightarrow \bar{N}_{3}(Z) \equiv \bar{C}_{\overline{i j k}} Z^{\bar{i}} Z^{\bar{j}} Z^{\bar{k}} .
$$

For an arbitrary SKG, it is in general hard to compute

$$
\frac{S_{B H}}{\pi}=\left.V_{B H}\right|_{\partial_{\phi} V_{B H}=0}=V_{B H}\left(\phi_{H}(\mathcal{P}), \mathcal{P}\right)
$$

where $\phi_{H}(\mathcal{P})$ are the horizon scalar configurations solving the Attractor Eqs. (5.8). However, the situation dramatically simplifies for symmetric SK manifolds

$$
\frac{G_{4}}{H_{4}}
$$

in which case a classification, analogous to the one available for $\mathcal{N}>2$-extended, $d=4$ supergravities (see e.g. [13] and Refs. therein; see also Sects. 3 and 4) can be performed [6].

In the treatment below, we are going to give a remarkable general topological formula for $V_{B H}\left(\phi_{H}(\mathcal{P}), \mathcal{P}\right)$ for symmetric SKG, which is manifestly invariant under diffeomorphisms of the SK scalar manifold, and which holds for any choice of symplectic basis of "bare" charges $\mathcal{P}$ and of special coordinates (see e.g. [22] and Refs. therein) of the SK manifold itself. Indeed, such a formula by no means does refer to special coordinates, which may not even exist for certain parametrizations of $\frac{G_{4}}{H_{4}}$ itself.

It should be pointed out that a general formula for the $G_{4}$-invariant $\mathcal{I}_{4, \mathcal{N}=2}$ is known for the so-called $d$-SK homogeneous symmetric manifolds [26], and it reads $(a=1, \ldots, n-1)$ [4]:

$$
\mathcal{I}_{4, \mathcal{N}=2}(\mathcal{P})=-\left(p^{0} q_{0}+p^{a} q_{a}\right)^{2}+4\left[q_{0} \mathcal{I}_{3, \mathcal{N}=2}(p)-p^{0} \mathcal{I}_{3, \mathcal{N}=2}(q)+\left\{\mathcal{I}_{3, \mathcal{N}=2}(p), \mathcal{I}_{3, \mathcal{N}=2}(q)\right\}\right]
$$

where

$$
\begin{aligned}
\mathcal{I}_{3, \mathcal{N}=2}(p) & \equiv \frac{1}{3 !} d_{a b c} p^{a} p^{b} p^{c} \\
\mathcal{I}_{3, \mathcal{N}=2}(q) & \equiv \frac{1}{3 !} d^{a b c} q_{a} q_{b} q_{c} \\
\left\{\mathcal{I}_{3, \mathcal{N}=2}(p), \mathcal{I}_{3, \mathcal{N}=2}(q)\right\} & \equiv \frac{\partial \mathcal{I}_{3, \mathcal{N}=2}(p)}{\partial p^{a}} \frac{\partial \mathcal{I}_{3, \mathcal{N}=2}(q)}{\partial q_{a}}
\end{aligned}
$$


in which the constant (number) rank-3 symmetric tensor $d_{a b c}$ has been introduced (and $d^{a b c}$ is its suitably defined completely contravariant form). However, such a formula holds for a particular symplectic basis (namely the one inherited from the $\mathcal{N}=2, d=5$ theory, i.e. the one of special coordinates), in which the holomorphic prepotential $F(X)$ of SKG can be written as

$$
F(X) \equiv \frac{1}{3 !} d_{a b c} \frac{X^{a} X^{b} X^{c}}{X^{0}}
$$

In such a symplectic basis, the manifest symmetry is the $d=5 U$-duality $G_{5}$, under which $G_{4}$ branches as $G_{4} \rightarrow G_{5} \times S O(1,1)$. Indeed, $\mathcal{I}_{3, \mathcal{N}=2}(p)$ and $\mathcal{I}_{3, \mathcal{N}=2}(q)$ are nothing but respectively the magnetic and electric invariants (both cubic in $\mathcal{P}$ ) of the relevant symplectic representations of $G_{5}$.

Eq. (5.21) excludes the so-called quadratic (or minimally coupled [42]) sequence of symmetric SK manifolds (particular complex Grassmannians)

$$
\frac{S U(1, n-1)}{S U(n-1) \times U(1)}, n \in \mathbb{N}
$$

(not upliftable to $d=5$ ), for which $F(X)$ is given by (in the symplectic basis exhibiting the maximal non-compact symmetry $S U(1, n-1))$

$$
F(X)=-\frac{i}{2}\left[\left(X^{0}\right)^{2}-\sum_{i=1}^{n-1}\left(X^{i}\right)^{2}\right]
$$

and the invariant of the symplectic representation of $G_{4}=S U(1, n-1)$ reads as follows (notice it is quadratic in $\mathcal{P}$ ) [29]:

$$
\mathcal{I}_{2, \mathcal{N}=2}(\mathcal{P})=\left(p^{0}\right)^{2}+q_{0}^{2}-\sum_{i=1}^{n-1}\left(\left(p^{i}\right)^{2}+q_{i}^{2}\right)=|Z|^{2}-g^{i j}\left(D_{i} Z\right) \bar{D}_{\bar{j}} \bar{Z}
$$

Due to the quadratic nature of the $G_{4}$-invariant $\mathcal{I}_{2, \mathcal{N}=2}(\mathcal{P})$ given by Eq. (5.28), the quadratic sequence of symmetric SK manifolds (5.26) exhibits only one small charge orbit, namely the lightlike one, beside the two large charge orbits determined in [6].

The symmetric SK manifolds whose geometry is determined by the holomorphic prepotential function (5.25) and the minimally coupled ones determined by Eq. (5.27) are all the possible symmetric SK manifolds. After [43], from the geometric perspective of SKG, symmetric SK manifolds can be characterized in the following way.

In SKG the Riemann tensor obeys to the following constraint (see e.g. [22] and Refs. therein):

$$
R_{i \bar{j} k \bar{l}}=-g_{i \bar{j}} g_{k \bar{l}}-g_{i \bar{l}} g_{k \bar{j}}+C_{i k m} \bar{C}_{\bar{l} \bar{n} \bar{n}} g^{m \bar{n}}
$$

The requirement that the manifold to be symmetric demands the Riemann to be covariantly constant:

$$
D_{m} R_{i \bar{j} k \bar{l}}=0
$$


Due to the SKG constraint (5.29) and to covariant holomorphicity of the $C$-tensor (expressed by Eq. (5.5) ), Eq. (5.30) generally implies (for non-vanishing $C_{i j k}$ )

$$
D_{l} C_{i j k}=D_{(l} C_{i) j k}=0,
$$

where in the last step Eq. (5.6) was used. Thus, in a SK symmetric space both the Riemann tensor and the $C$-tensor are covariantly constant. Eq. (5.31) implies the following relation [6]

$$
C_{j(l m} C_{p q) k} \bar{C}_{\overline{i j k} \bar{k}} g^{j \bar{j}} g^{k \bar{k}}=\frac{4}{3} C_{(l m p} g_{q) \bar{i}}
$$

which is nothing but the "dressed" form of the analogous relation holding for the $d$-tensor itself [44, 43]

$$
d_{j(l m} d_{p q) k} d^{i j k}=\frac{4}{3} d_{(l m p} \delta_{q)}^{i} .
$$

The quadratic sequence of symmetric manifolds (5.26) whose SKG is determined by the prepotential (5.27) has

$$
C_{i j k}=0,
$$

whereas the remaning symmetric SK manifolds, whose prepotential in the special coordinates is given by Eq. (5.25) (with $d_{a b c}$ constrained by Eq. (5.33)), correspond to

$$
C_{a b c}=e^{K} d_{a b c} .
$$

By using Eqs. (5.31) and (5.32), as well as the SKG identities (5.2)-(5.4) (which, for symmetric SKG, are equivalent to the Maurer-Cartan Eqs., as Eqs. (3.3) and (4.4)-(4.5) for $\mathcal{N}=8$ and $\mathcal{N}=4, d=4$ supergravities, respectively; see e.g. [29, 21]), one can prove that the following quartic expression is a duality invariant for all symmetric SK manifolds :

$$
\begin{aligned}
\mathcal{I}_{4, \mathcal{N}=2, \text { symm }}(\phi, \mathcal{P})= & \left(Z \bar{Z}-Z_{i} \bar{Z}^{i}\right)^{2}+ \\
& +\frac{2}{3} i\left(Z N_{3}(\bar{Z})-\overline{Z N}_{3}(Z)\right)+ \\
& -g^{i \bar{i}} C_{i j k} \bar{C}_{\bar{i} \bar{l} \bar{m}} \bar{Z}^{j} \bar{Z}^{k} Z^{\bar{l}} Z^{\bar{m}}
\end{aligned}
$$

where the matter charges have been re-noted as $Z_{i} \equiv D_{i} Z, Z^{\bar{i}}=g^{j \bar{i}} Z_{j}$, and definition (5.18) was recalled.

As claimed above, $\mathcal{I}_{4, \mathcal{N}=2, \text { symm }}$ given by Eq. (5.36) is $\phi$-dependent only apparently, i.e. it is topological, merely charge-dependent:

$$
\frac{\partial \mathcal{I}_{4, \mathcal{N}=2, \text { symm }}(\phi, \mathcal{P})}{\partial \phi}=0 \Leftrightarrow \mathcal{I}_{4, \mathcal{N}=2, \text { symm }}=\mathcal{I}_{4, \mathcal{N}=2, \text { symm }}(\mathcal{P})
$$

Thus, by recalling Eq. (1.5), the general entropy-area formula [8] for extremal BHs in $\mathcal{N}=2$, $d=4$ supergravity coupled to Abelian vector multiplets whose scalar manifold is a symmetric (SK) space reads as follows:

$$
\frac{S_{B H}}{\pi}=\left.V_{B H}\right|_{\partial_{\phi} V_{B H}=0}=V_{B H}\left(\phi_{H}(\mathcal{P}), \mathcal{P}\right)=\left|\mathcal{I}_{4, \mathcal{N}=2, \text { symm }}(\mathcal{P})\right|^{1 / 2}
$$


Let us briefly analyze Eq. (5.36).

As for the case of $\mathcal{N}=8, d=4$ supergravity treated in Sect. 3, one can introduce a phase $\vartheta$ as follows (recall definitions (5.18)):

$$
e^{2 i \vartheta} \equiv-\frac{Z N_{3}(\bar{Z})}{\overline{Z N}_{3}(Z)}=\frac{i Z C_{i j k} \bar{Z}^{i} \bar{Z}^{j} \bar{Z}^{k}}{-i \bar{C}_{\bar{l} \overline{m n}} Z^{\bar{l}} Z^{\bar{m}} Z^{\bar{n}}}
$$

Thus, $\vartheta$ is the phase of the quantity $i Z N_{3}(\bar{Z}): \vartheta \equiv \vartheta_{i Z N_{3}(\bar{Z})}$. It is then immediate to compute $\vartheta$ from Eq. (5.36):

$$
\cos \vartheta(\phi, \mathcal{P})=\frac{3\left[\mathcal{I}_{4, \mathcal{N}=2, \text { symm }}(\mathcal{P})-\left(Z \bar{Z}-Z_{i} \bar{Z}^{i}\right)^{2}+g^{i \bar{i}} C_{i j k} \bar{C}_{\bar{i} \bar{l} \bar{m}} \bar{Z}^{j} \bar{Z}^{k} Z^{\bar{l}} Z^{\bar{m}}\right]}{2^{2}\left|Z N_{3}(\bar{Z})\right|} .
$$

Notice that through Eq. (5.40) $(\cos ) \vartheta$ is determined in terms of the scalar fields $\phi$ and of the $\mathrm{BH}$ charges $\mathcal{P}$, also along the small orbits where $\mathcal{I}_{4, \mathcal{N}=2, \text { symm }}=0$. However, Eq. (5.40) is not defined in the cases in which $Z N_{3}(\bar{Z})=0$. In such cases, $\vartheta$ is actually undetermined. It should be clearly pointed out that the phase $\vartheta$ has nothing to do with the phase of the $U(1)$ bundle over the SK-Hodge vector multiplets' scalar manifold (see e.g. [22] and Refs. therein).

1. For $\frac{1}{2}$-BPS attractors (defined by the constraints (5.9)), Eq. 5.36 yields

$$
\left.\mathcal{I}_{4, \mathcal{N}=2, \text { symm }}\right|_{\frac{1}{2}-B P S}=(Z \bar{Z})_{\frac{1}{2}-B P S}^{2}=|Z|_{\frac{1}{2}-B P S}^{4},
$$

as in turn also implied by Eqs. (5.10) and (1.5) (or equivalently (5.38)). Notice that Eqs. (5.10) and (5.41) are general, i.e. they hold for any SKG, regardless the symmetric nature of the SK vector multiplets' scalar manifold. Furthermore, the constraints (5.9) imply that at the event horizon of $\frac{1}{2}$-BPS extremal BHs it holds

$$
\left[N_{3}(\bar{Z})\right]_{\frac{1}{2}-B P S}=0 \Rightarrow \vartheta_{\frac{1}{2}-B P S} \text { undetermined. }
$$

2. For non-BPS $Z=0$ attractors (defined by the constraints 5.13 which, through Eqs. (5.8), imply Eq. (5.14)), Eq. (5.36) yields

$$
\left.\mathcal{I}_{4, \mathcal{N}=2, \text { symm }}\right|_{\text {non }-B P S, Z=0}=\left(Z_{i} \bar{Z}^{i}\right)_{n o n-B P S, Z=0}^{2}=\left[g^{i \bar{j}}\left(\partial_{i} Z\right) \bar{\partial}_{\bar{j}} \bar{Z}_{n o n-B P S, Z=0}^{2} .\right.
$$

Notice that Eqs. (5.15) and (5.43) are general, i.e. they hold for any SKG, regardless the symmetric nature of the SK vector multiplets' scalar manifold. Furthermore, the constraints (5.9) imply that at the event horizon of non-BPS $Z=0$ extremal BHs it holds

$$
Z_{\text {non-BPS,Z=0 }}=0 \Rightarrow \vartheta_{\text {non-BPS,Z=0 }} \text { undetermined }
$$


3. For non-BPS $Z \neq 0$ attractors (defined by the constraints (5.16) as well as by Eqs. (5.8), Eqs. (5.36) and (5.17) yield

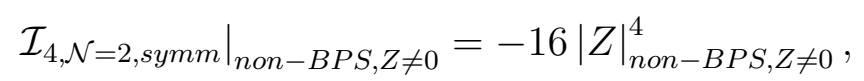

thus implying, through Eq. (5.7) [45, 30, 6, 13]

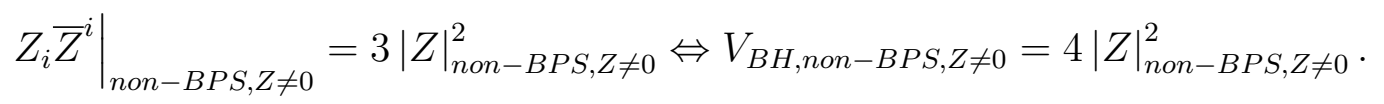

By plugging Eqs. (5.8), (5.16), (5.17) and (5.45) into Eq. (5.40), it follows that at the event horizon of non-BPS $Z \neq 0$ extremal $\mathrm{BHs}$ it holds that

$$
\vartheta_{\text {non }-B P S, Z \neq 0}=\pi+2 k \pi, k \in \mathbb{Z} .
$$

It should be remarked that, differently from the results (5.10)-(5.12), (5.41)-(5.42) (holding for $\frac{1}{2}$-BPS attractors) and from the results 5.14 - (5.15), (5.43)-(5.44) (holding for non-BPS $Z=0$ attractors), Eqs. (5.45)-(5.47) are not general: i.e. they hold at the event horizon of extremal non-BPS $Z \neq 0$ BHs for symmetric SK manifolds, but they do not hold true for generic SKG. However, when going beyond the symmetric SK case (and thus encompassing both homogeneous non-symmetric [26, 46] and non-homogeneous SK spaces), one can compute both $V_{B H, n o n-B P S, Z \neq 0}$ and $\left.\mathcal{I}_{4, \mathcal{N}=2, \text { symm }}\right|_{\text {non-BPS,Z } \neq 0}$, and express the deviation from the symmetric case considered above in terms of the complex quantity [13]

$$
\Delta \equiv-\frac{3}{4} \frac{E_{i \bar{j} k l \bar{m}} \bar{Z}^{i} Z^{\bar{j}} Z^{\bar{k}} Z^{\bar{l}} Z^{\bar{m}}}{\bar{N}_{3}(Z)}
$$

where the tensor $E_{i j \overline{k l} \bar{m}}$ was firstly introduced in [26] (see also [13]). The results of straightforward computations read as follows:

$$
\begin{aligned}
& V_{B H, n o n-B P S, Z \neq 0}=4|Z|_{n o n-B P S, Z \neq 0}^{2}+\Delta_{n o n-B P S, Z \neq 0}
\end{aligned}
$$

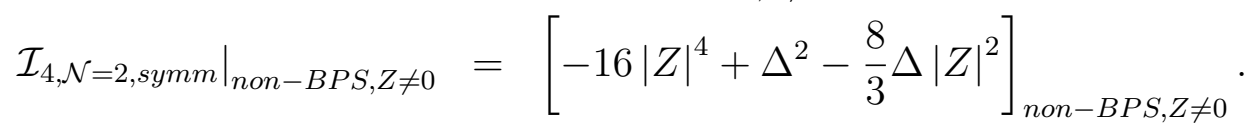

Notice that, as yielded e.g. by Eq. (5.49), $\Delta$ is real at the non-BPS $Z \neq 0$ critical points of $V_{B H}$. For symmetric SK manifolds $E_{i \overline{j k l} \bar{m}}=0$ globally, and thus Eqs. $(5.49)$ and (5.50) respectively reduce to Eqs. (5.46) and (5.45). On the other hand, the results $5.45)-(5.46)$ hold also for those non-symmetry SK spaces $\left(E_{i \overline{j k l} \bar{m}} \neq 0\right)$ such that

$$
\Delta_{n o n-B P S, Z \neq 0}=0 \Leftrightarrow\left(E_{i \overline{j k l} \bar{m}} \bar{Z}^{i} Z^{\bar{j}} Z^{\bar{k}} Z^{\bar{l}} Z^{\bar{m}}\right)_{n o n-B P S, Z \neq 0}
$$

where in the implication " $\Rightarrow$ " the assumption $\left[\bar{N}_{3}(Z)\right]_{n o n-B P S, Z \neq 0} \neq 0$ was made. The condition (5.51) might explain some results obtained for generic $(d-$ )SKGs in some particular supporting $\mathrm{BH}$ charge configurations in [45] (see also the treatment in [13] and [39]). 
Consistently, for the quadratic minimally coupled sequence (5.26), for which Eq. (5.34) holds, Eq. (5.36) formally reduces to

$$
\begin{gathered}
\left.\mathcal{I}_{4, \mathcal{N}=2, \text { symm }}\right|_{C_{i j k}=0}=\left(Z \bar{Z}-Z_{i} \bar{Z}^{i}\right)^{2} ; \\
\mathbb{1} \\
\left.\left|\mathcal{I}_{4, \mathcal{N}=2, \text { symm }}\right|_{C_{i j k}=0}\right|^{1 / 2}=\left|\mathcal{I}_{2, \mathcal{N}=2}\right|,
\end{gathered}
$$

where $\mathcal{I}_{2, \mathcal{N}=2}$ is given by Eq. 5.28).

Remarkably, Eq. (5.36) turns out to be directly related to the quantity $-h$ given by Eq. (2.31) of [26] (see also the treatment of [47]). This is seen by noticing that Eq. (4.42) of [26] coincides with Eq. (5.21) (along with definitions (5.22)-(5.24)). Note that the mapping of quaternionic coordinates $\left(A^{\Lambda}, B_{\Lambda}\right)^{T}$ into the charges $\mathcal{P}^{T}=\left(p^{\Lambda}, q_{\Lambda}\right)^{T}$ (in special coordinates) is related to the $d=3$ attractor flows (see e.g. [48, 49, 50]).

For symmetric SK manifolds, small charge orbits of the symplectic representation of $G_{4}$ are known to exist since [4] and [5].

- small lightlike charge orbits are defined by the $G_{4}$-invariant constraint

$$
\begin{gathered}
\mathcal{I}_{4, \mathcal{N}=2, \text { symm }}=0 \\
\mathbb{\Downarrow} \\
\left(Z \bar{Z}-Z_{i} \bar{Z}^{i}\right)^{2}+\frac{2}{3} i\left(Z N_{3}(\bar{Z})-\overline{Z N}_{3}(Z)\right)=g^{i \bar{i}} C_{i j k} \bar{C}_{\bar{i} \bar{l} \bar{m}} \bar{Z}^{j} \bar{Z}^{k} Z^{\bar{l}} Z^{\bar{m}} .
\end{gathered}
$$

In this case, Eq. (5.40) reduces to

$$
\left.\cos \vartheta(\phi, \mathcal{P})\right|_{\mathcal{I}_{4, \mathcal{N}=2, s y m m}=0}=-\left.\frac{3\left[\left(Z \bar{Z}-Z_{i} \bar{Z}^{i}\right)^{2}-g^{i \bar{i}} C_{i j k} \bar{C}_{\bar{i} \bar{m} \bar{Z}} \bar{Z}^{j} \bar{Z}^{k} Z^{\bar{l}} Z^{\bar{m}}\right]}{2^{2}\left|Z N_{3}(\bar{Z})\right|}\right|_{\substack{\mathcal{I}_{4, \mathcal{N}=2, \text { symm }}=0 \\(5,55)}} .
$$

- Beside the constraint (5.53)-(5.54), small critical charge orbits are defined by the following $G_{4}$-invariant set of first order differential constraints, as well:

$$
\frac{\partial \mathcal{I}_{4, \mathcal{N}=2, \text { symm }}}{\partial Z}=0=\frac{\partial \mathcal{I}_{4, \mathcal{N}=2, \text { symm }}}{\partial Z_{i}} .
$$

- Beside the constraints (5.53)-(5.54) and (5.56), small doubly-critical charge orbits are also defined by the following set of second order differential constraints, as well:

$$
\mathcal{D}_{i j} \mathcal{I}_{4, \mathcal{N}=2, \text { symm }}=0=\mathcal{D}_{i} \mathcal{I}_{4, \mathcal{N}=2, \text { symm }},
$$


where the second-order differential operators $\mathcal{D}_{i \bar{j}}$ and $\mathcal{D}_{i}$ have been introduced:

$$
\begin{aligned}
\mathcal{D}_{i \bar{j}} & \equiv R_{i \bar{j} k}^{l} \frac{\partial}{\partial Z_{k}} \frac{\partial}{\overline{\partial Z}^{l}} \\
\mathcal{D}_{i} & \equiv C_{i j k} \frac{\partial}{\partial Z_{j}} \frac{\partial}{\partial Z_{k}}
\end{aligned}
$$

Notice that, through the definitions $(5.58)$ and (5.59), the constraints $(5.57)$ are $G_{4^{-}}$ invariant, because they are equivalent to the following constraint:

$$
\left.\frac{\partial^{2} \mathcal{I}_{4, \mathcal{N}=2, \text { symm }}}{\partial Z_{\text {sympl }\left(G_{4}\right)} \partial Z_{\text {sympl }\left(G_{4}\right)}}\right|_{\operatorname{Adj}\left(G_{4}\right)}=0
$$

where

$$
Z_{\text {sympl }\left(G_{4}\right)} \equiv\left(Z, \bar{Z}_{\bar{i}}, \bar{Z}, Z_{i}\right)^{T},
$$

and the change of charge basis between the manifestly $H_{4}$-covariant (in "flat" local coordinates) basis $Z_{\mathbf{s y m p l}\left(G_{4}\right)}$ and the manifestly $S p(2 n, \mathbb{R})$-covariant basis $\mathcal{P}$ (defined by Eq. (1.2) ) is expressed by the fundamental identities of the SKG (see e.g. [51, 22] and Refs. therein). Indeed, by considering the Cartan decomposition of the Lie algebra of $G_{4}$ :

$$
\mathfrak{g}_{4}=\mathfrak{h}_{4}+\mathfrak{k}_{4},
$$

and switching to "flat" local coordinates in the scalar manifold (here denoted by capital Latin indices), it holds that $\mathcal{D}_{I}$ ( "flat" version of the operator defined in Eq. (5.59) ) is $\mathfrak{k}_{4}$-valued. Furthermore, in symmetric manifolds $R_{I \bar{J} K}{ }^{L}$ is a two-form (in the first two "flat" local indices) which is Lie algebra-valued in $\mathfrak{h}_{4}$, and thus $\mathcal{D}_{I \bar{J}}$ ( "flat" version of the operator defined in Eq. (5.58)) turns out to be $\mathfrak{h}_{4}$-valued. Notice that Eq. (5.60), $G_{4}$-invariantly defining the small doubly-critical charge orbit(s) of the $\mathcal{N}=2, d=4$ vector multiplets' symmetric SK scalar manifolds, is the analogue of Eq. (3.42), which defines in an $E_{7(7)}$-invariant way the small doubly-critical charge orbit of $\mathcal{N}=8, d=4$ pure supergravity. It should be also recalled that in $\mathcal{N}=4, d=4$ matter coupled supergravity small doubly-critical (or higher-order-critical) charge orbits (independent from the small critical ones) are absent. As treated in Sect. 4, all small critical charge orbits of the $\mathcal{N}=4$ theory actually are doubly-critical, and the analogues of Eqs. (3.42) and (5.60) are given, through Eq. (4.50) and definitions (4.51) and (4.53), by the rich case study exhibited by Eqs. (4.48)-(4.49) and) 4.56)-4.57).

The classification of small charge orbits of the relevant symplectic representation of $G_{4}$ for $\mathcal{N}=2, d=4$ supergravity coupled to Abelian vector multiplets whose scalar manifold $\frac{G_{4}}{H_{4}}$ is (SK) symmetric, performed in accordance to their "order of criticality" (lightlike, critical, doubly-critical), will be given elsewhere. 


\section{ADM Mass for BPS Extremal Black Hole States}

For BPS BH states in $d=4$ ungauged 8 supergravity theories, the ADM mass [27] $M_{A D M}\left(\phi_{\infty}, \mathcal{P}\right)$ is defined as the largest (of the absolute values) of the skew-eigenvalues of the (spatially asymptotically) central charge matrix $Z_{A B}\left(\phi_{\infty}, \mathcal{P}\right)$ which saturate the BPS bound (2.28). The skew-diagonalization of $Z_{A B}$ is made by performing a suitable transformation of the $\mathcal{R}$-symmetry, and thus by going to the so-called normal frame. In such a frame, the skeweigenvalues of $Z_{A B}$ can be taken to be real and positive (up to an eventual overall phase). By saturating the BPS bound (2.28), it therefore holds that

$$
M_{A D M}\left(\phi_{\infty}, \mathcal{P}\right)=\left|\mathbf{Z}_{1}\left(\phi_{\infty}, \mathcal{P}\right)\right| \geqslant \ldots \geqslant\left|\mathbf{Z}_{[\mathcal{N} / 2]}\left(\phi_{\infty}, \mathcal{P}\right)\right|
$$

where $\mathbf{Z}_{1}(\phi, \mathcal{P}), \ldots, \mathbf{Z}_{[\mathcal{N} / 2]}(\phi, \mathcal{P})$ denote the set of skew-eigenvalues of $Z_{A B}(\phi, \mathcal{P})$, and square brackets denote the integer part of the enclosed number. As mentioned at the end of Sect. 2 , if $1 \leqslant \mathbf{k} \leqslant[\mathcal{N} / 2]$ of the bounds expressed by Eq. (2.28) are saturated, the corresponding extremal BH state is named to be $\frac{\mathbf{k}}{\mathcal{N}^{-}}$BPS. Thus, the minimal fraction of total supersymmetries (pertaning to the asymptotically flat space-time metric) preserved by the extremal $\mathrm{BH}$ background within the considered assumptions is $\frac{1}{\mathcal{N}}$ (for $\mathbf{k}=1$ ), while the maximal one is $\frac{1}{2}$ $\left(\right.$ for $\left.\mathbf{k}=\frac{\mathcal{N}}{2}\right)$.

The ADM mass and its symmetries are different, depending on $\mathbf{k}$.

\section{1 $\mathcal{N}=8$}

In $\mathcal{N}=8, d=4$ supergravity (treated in Sect. 3), the $E_{7(7)} U$-duality symmetry only allows the cases [3] $\mathbf{k}=1,2,4$. By recalling the review given in Sect. 3, the maximal compact symmetries of the supporting charge orbits respectively read [3, 4, 30, 13, 32, 33 .

$$
\begin{aligned}
& \mathbf{k}=1: S U(2) \times S U(6) ; \\
& \mathbf{k}=2: U S p(4) \times S U(4) \\
& \mathbf{k}=4: U S p(8)
\end{aligned}
$$

and they hold all along the respective scalar flows. While cases $\mathbf{k}=2$ and 4 are small (thus not enjoying the attractor mechanism), case $\mathbf{k}=1$ can be either large or small.

In the large $\mathbf{k}=1$ case, the attractor mechanism makes the maximal compact symmetry $S U(2) \times S U(6)$ of the supporting charge orbit $\mathcal{O}_{\frac{1}{8}-B P S \text {, large }}$ fully manifest as a symmetry of the central charge matrix $Z_{A B}$ through the symmetry enhancement (3.17) at the event horizon of the considered extremal $\mathrm{BH}$.

Furthermore, the $\frac{1}{4}$-BPS saturation of the $\mathcal{N}=8$ BPS bound (all along the $\frac{1}{4}$-BPS scalar flow) has the following peculiar structure (recall Eq. (3.35) [3]

$$
\left|\mathbf{Z}_{1}(\phi, \mathcal{P})\right|=\left|\mathbf{Z}_{2}(\phi, \mathcal{P})\right|>\left|\mathbf{Z}_{3}(\phi, \mathcal{P})\right|=\left|\mathbf{Z}_{4}(\phi, \mathcal{P})\right|
$$

\footnotetext{
${ }^{8}$ In the present paper only ungauged supergravities are treated. It is here worth remarking that the definition of the ADM mass for (eventually rotating) asymptotically non-flat black holes in gauged supergravities is a fairly subtle issue, addressed by various studies in literature (see e.g. [52, 53], and Refs. therein).
} 
where it should be recalled that in Sect. 3 the notation $e_{i} \equiv\left|\mathbf{Z}_{i}\right|(i=1, \ldots, 4)$ was used.

As done in Sect. 3, let us denote with $\lambda_{i}(i=1, \ldots, 4)$ the four real non-negative eigenvalues of the $8 \times 8$ Hermitian matrix $Z_{A B} \bar{Z}^{C B}=\left(Z Z^{\dagger}\right)_{A}^{C} \equiv A_{A}^{C}$. Their relation with the absolute values of the complex skew-eigenvalues $e_{i}$ of $Z_{A B}$ is given by Eq. (3.29). As mentioned, the ordering $\lambda_{1} \geqslant \lambda_{2} \geqslant \lambda_{3} \geqslant \lambda_{4}$ does not imply any loss of generality. After [9] (see in particular Eqs. (4.74), (4.75), (4.86) and (4.87) therein), the explicit expression of $\lambda_{i}$ in terms of $U(8)$-invariants (namely of $\operatorname{Tr} A, \operatorname{Tr}\left(A^{2}\right), \operatorname{Tr}\left(A^{3}\right)$ and $\operatorname{Tr}\left(A^{4}\right)$, and suitable powers) is known, and it can be thus be used in order to compute the ADM mass of $\frac{\mathbf{k}}{8}$-BPS extremal $\mathrm{BH}$ states of $\mathcal{N}=8, d=4$ supergravity.

The $\lambda_{i}$ 's are solution of the (square root of) characteristic equation [9]

$$
\sqrt{\operatorname{det}(A-\lambda \mathbb{I})}=\prod_{i=1}^{4}\left(\lambda-\lambda_{i}\right)=\lambda^{4}+a \lambda^{3}+b \lambda^{2}+c \lambda+d=0,
$$

where 9

$$
\begin{aligned}
a & \equiv-\frac{1}{2} \operatorname{Tr} A=-\left(\lambda_{1}+\lambda_{2}+\lambda_{3}+\lambda_{4}\right) \\
b & \equiv \frac{1}{4}\left[\frac{1}{2}(\operatorname{Tr} A)^{2}-\operatorname{Tr}\left(A^{2}\right)\right]= \\
& =\lambda_{1} \lambda_{2}+\lambda_{1} \lambda_{3}+\lambda_{1} \lambda_{4}+\lambda_{2} \lambda_{3}+\lambda_{2} \lambda_{4}+\lambda_{3} \lambda_{4} ; \\
c & \equiv-\frac{1}{6}\left[\frac{1}{8}(\operatorname{Tr} A)^{3}+\operatorname{Tr}\left(A^{3}\right)-\frac{3}{4} \operatorname{Tr}\left(A^{2}\right) \operatorname{Tr} A\right]= \\
& =-\left(\lambda_{1} \lambda_{2} \lambda_{3}+\lambda_{1} \lambda_{2} \lambda_{4}+\lambda_{1} \lambda_{3} \lambda_{4}+\lambda_{2} \lambda_{3} \lambda_{4}\right) ; \\
d & \equiv \frac{1}{4}\left[\frac{1}{96}(\operatorname{Tr} A)^{4}+\frac{1}{8} \operatorname{Tr}{ }^{2}\left(A^{2}\right)+\frac{1}{3} \operatorname{Tr}\left(A^{3}\right) \operatorname{Tr} A+\right] \\
& =\sqrt{\operatorname{det} A}=\lambda_{1} \lambda_{2} \lambda_{3} \lambda_{4} .
\end{aligned}
$$

The system (6.7)- 6.10 can be inverted, yielding

$$
\begin{aligned}
& \lambda_{1,2}=-\frac{a}{4}+\frac{s}{2} \pm \frac{1}{2} \sqrt{\frac{a^{2}}{2}-\frac{4 b}{3}-\frac{\left(a^{3}-4 a b+8 c\right)}{4 s}-\frac{u}{3 w}-\frac{w}{3}} \\
& \lambda_{3,4}=-\frac{a}{4}-\frac{s}{2} \pm \frac{1}{2} \sqrt{\frac{a^{2}}{2}-\frac{4 b}{3}+\frac{\left(a^{3}-4 a b+8 c\right)}{4 s}-\frac{u}{3 w}-\frac{w}{3}}
\end{aligned}
$$


where

$$
\begin{aligned}
u & \equiv b^{2}+12 d-3 a c ; \\
v & \equiv 2 b^{3}+27 c^{2}-72 b d-9 a b c+27 a^{2} d ; \\
w & \equiv\left(\frac{v+\sqrt{v^{2}-4 u^{3}}}{2}\right)^{1 / 3} ; \\
s & \equiv \sqrt{\frac{a^{2}}{4}-\frac{2 b}{3}+\frac{u}{3 w}+\frac{w}{3}} .
\end{aligned}
$$

Notice that the positivity of quantities under square root in Eqs. 6.11), (6.12), 6.15) and (6.16) always holds. Furthermore, Eq. (6.6) is at most of fourth order (for $\mathbf{k}=1$ ), of second order for $\mathbf{k}=2$, and of first order for $\mathbf{k}=1$.

1. $\mathbf{k}=1\left(\frac{1}{8}\right.$-BPS, either large or small). The $\frac{1}{8}$-BPS extremal BH square ADM mass is

$$
M_{A D M, \frac{1}{8}-B P S}^{2}\left(\phi_{\infty}, \mathcal{P}\right)=\lambda_{1}\left(\phi_{\infty}, \mathcal{P}\right)
$$

where $\lambda_{1}\left(>\lambda_{2}>\lambda_{3}>\lambda_{4}\right.$, since $a<0$ and $\left.s>0\right)$ is given by Eq. (6.11). In the large $\mathbf{k}=1$ case $\lambda_{2}=\lambda_{3}=\lambda_{4}=0$ at the event horizon of the extremal $\mathrm{BH}$, as given by Eq. (3.16).

2. $\mathbf{k}=2\left(\frac{1}{4}\right.$-BPS, small). As given by Eq. 3.35$)$, the eigenvalues are equal in pairs. By suitably renaming the two non-coinciding $\lambda$ 's, one gets

$$
\lambda_{1,2}=\frac{1}{8} \operatorname{Tr} A \pm \frac{1}{2} \sqrt{\frac{1}{2} \operatorname{Tr}\left(A^{2}\right)-\frac{1}{16}(\operatorname{Tr} A)^{2}} .
$$

As mentioned above, the maximal (compact) symmetry is manifest when $\lambda_{2}$ (in the renaming of Eq. (6.18) vanishes (see treatment in Sect. 3). Eq. (3.35) implies [9]

$$
\begin{aligned}
& c=\frac{1}{2} a\left(b-\frac{1}{4} a^{2}\right) ; \\
& d=\frac{1}{4}\left(b-\frac{1}{4} a^{2}\right)^{2} .
\end{aligned}
$$

In 9 Eqs. (6.19)-(6.20) were shown to be consequences of the criticality constraints (3.34. Thus, the $\frac{1}{4}$-BPS extremal BH square ADM mass is

$$
M_{A D M, \frac{1}{4}-B P S}^{2}\left(\phi_{\infty}, \mathcal{P}\right)=\lambda_{1}\left(\phi_{\infty}, \mathcal{P}\right)
$$

where $\lambda_{1}\left(>\lambda_{2}\right)$ is given by Eq. (6.18):

$$
M_{A D M, \frac{1}{4}-B P S}^{2}\left(\phi_{\infty}, \mathcal{P}\right)=\frac{1}{8} \operatorname{Tr} A\left(\phi_{\infty}, \mathcal{P}\right)+\frac{1}{2} \sqrt{\frac{1}{2} \operatorname{Tr}\left(A^{2}\right)\left(\phi_{\infty}, \mathcal{P}\right)-\frac{1}{16}\left(\operatorname{Tr} A\left(\phi_{\infty}, \mathcal{P}\right)\right)^{2}} .
$$


3. $\mathbf{k}=4\left(\frac{1}{2}\right.$-BPS, small). This case can be obtained from the $\frac{1}{4}$-BPS considered at point 2 by further putting $\lambda_{1}=\lambda_{2}$ in Eq. 6.18). Thus, all eigenvalues of the Hermitian $8 \times 8$ matrix $A$ are equal:

$$
A_{A}^{C}=\frac{1}{8}(\operatorname{Tr} A) \delta_{A}^{C},
$$

which implies

$$
\operatorname{Tr}\left(A^{2}\right)=\frac{1}{8}(\operatorname{Tr} A)^{2} .
$$

Therefore, $\frac{1}{2}$-BPS extremal BH square ADM mass is given by

$$
M_{A D M, \frac{1}{2}-B P S}^{2}\left(\phi_{\infty}, \mathcal{P}\right)=\frac{1}{8} \operatorname{Tr} A\left(\phi_{\infty}, \mathcal{P}\right)=\frac{1}{16} Z_{A B}\left(\phi_{\infty}, \mathcal{P}\right) \bar{Z}^{A B}\left(\phi_{\infty}, \mathcal{P}\right) .
$$

\section{$6.2 \mathcal{N}=4$}

In $\mathcal{N}=4, d=4$ supergravity (treated in Sect. 4), the $S L(2, \mathbb{R}) \times S O(6, M) U$-duality symmetry only allows the cases [3] $\mathbf{k}=1,2$. By recalling the treatment of Sect. 4, the respective maximal compact symmetries read [3, 4, 13, 39]

$$
\begin{aligned}
& \mathbf{k}=1:(S U(2))^{2} \times S O(M) \times S O(2) ; \\
& \mathbf{k}=2: U S p(4) \times S O(M),
\end{aligned}
$$

and they hold all along the respective scalar flows. While case $\mathbf{k}=1$ is large, case $\mathbf{k}=2$ is small (thus not enjoying the attractor mechanism).

In the large $\mathbf{k}=1$ case, the attractor mechanism makes the maximal compact symmetry $(S U(2))^{2} \times S O(M) \times S O(2)$ of the supporting charge orbit $\mathcal{O}_{\frac{1}{4}-B P S \text {, large }}$ fully manifest as a symmetry of the central charge matrix $Z_{A B}$ through the symmetry enhancement (recall Eq. (4.25)

$$
(S U(2))^{2} \times S O(M-2) \times S O(2) \stackrel{r \rightarrow r_{H}^{+}}{\longrightarrow}(S U(2))^{2} \times S O(M) \times S O(2)
$$

at the event horizon of the considered extremal BH.

As done in Sect. 4 and in the treatment of case $\mathcal{N}=8, d=4$ above, let us denote with $\lambda_{1}$ and $\lambda_{2}$ the two real non-negative eigenvalues of the $4 \times 4$ Hermitian matrix $Z_{A B} \bar{Z}^{C B}=$ $\left(Z Z^{\dagger}\right)_{A}^{C} \equiv A_{A}^{C}$. Their relation with the absolute values of the complex skew-eigenvalues $e_{i}$ of $Z_{A B}$ is given by Eq. (3.29). As mentioned, the ordering $\lambda_{1} \geqslant \lambda_{2}$ does not imply any loss of generality. After [9], the explicit expression of $\lambda_{1}$ and $\lambda_{2}$ in terms of $(U(4) \times S O(M))$ invariants (namely of $\operatorname{Tr} A, \operatorname{Tr}\left(A^{2}\right)$ and $(\operatorname{Tr} A)^{2}$ ) is known, and it can be thus be used in order to compute the ADM mass of $\frac{\mathbf{k}}{4}$-BPS extremal BH states of $\mathcal{N}=4, d=4$ supergravity.

Indeed, $\lambda_{1}$ and $\lambda_{2}$ are solution of the (square root of) characteristic equation [9]

$$
\sqrt{\operatorname{det}(A-\lambda \mathbb{I})}=\prod_{i=1}^{2}\left(\lambda-\lambda_{i}\right)=\lambda^{2}-\frac{1}{2}(\operatorname{Tr} A) \lambda+(\operatorname{det} A)^{1 / 2}=0,
$$


whose solution reads

$$
\lambda_{1,2}=\frac{1}{2}\left(\frac{1}{2} \operatorname{Tr} A \pm \sqrt{\operatorname{Tr}\left(A^{2}\right)-\frac{1}{4}(\operatorname{Tr} A)^{2}}\right) .
$$

Notice that the positivity of quantities under square root in Eq. (6.30) always holds. Furthermore, Eq. (6.29) is at most of second order (for $\mathbf{k}=1$ ) and of first order for $\mathbf{k}=2$.

1. $\mathbf{k}=1$ ( $\frac{1}{4}$-BPS large). The $\frac{1}{4}$-BPS extremal BH square ADM mass is

$$
\begin{aligned}
M_{A D M, \frac{1}{4}-B P S}^{2}\left(\phi_{\infty}, \mathcal{P}\right) & =\lambda_{1}\left(\phi_{\infty}, \mathcal{P}\right)= \\
& =\frac{1}{2}\left(\frac{1}{2} \operatorname{Tr} A\left(\phi_{\infty}, \mathcal{P}\right)+\sqrt{\operatorname{Tr}\left(A^{2}\right)\left(\phi_{\infty}, \mathcal{P}\right)-\frac{1}{4}\left(\operatorname{Tr} A\left(\phi_{\infty}, \mathcal{P}\right)\right)^{2}}\right)
\end{aligned}
$$

where $\lambda_{1}>\lambda_{2}$. Notice that $\lambda_{2}=0$ at the event horizon of the extremal BH, as given by Eq. 4.23.

2. $\mathbf{k}=2$ ( $\frac{1}{2}$-BPS, small). This case can be obtained from the $\frac{1}{4}$-BPS considered at point 1 by further putting $\lambda_{1}=\lambda_{2}$ in Eq. 6.30. Thus, all eigenvalues of the Hermitian $4 \times 4$ matrix $A$ are equal:

$$
A_{A}^{C}=\frac{1}{4}(\operatorname{Tr} A) \delta_{A}^{C},
$$

which implies

$$
\operatorname{Tr}\left(A^{2}\right)=\frac{1}{4}(\operatorname{Tr} A)^{2} .
$$

Thus, the $\frac{1}{2}$-BPS extremal BH square ADM mass is

$$
M_{A D M, \frac{1}{2}-B P S}^{2}\left(\phi_{\infty}, \mathcal{P}\right)=\lambda_{1}\left(\phi_{\infty}, \mathcal{P}\right)=\lambda_{2}\left(\phi_{\infty}, \mathcal{P}\right)=\frac{1}{4} \operatorname{Tr} A\left(\phi_{\infty}, \mathcal{P}\right)
$$

It should be here remarked that the $\mathcal{R}$-symmetry of the $\frac{\mathrm{k}}{\mathcal{N}}$-BPS extremal BH states, i.e. the compact symmetry of the solution in the normal frame (determining the automorphism group of the supersymmetry algebra in the rest frame) gets broken as follows:

$$
\mathcal{R} \longrightarrow U S p(2 \mathbf{k}) \times \ldots
$$

This is precisely the symmetry of the $\frac{\mathbf{k}}{\mathcal{N}}$-BPS saturated massive multiplets of the $\mathcal{N}$-extended, $d=4$ Poincaré supersymmetry algebra [54].

We end this Section by finally commenting about the ADM mass for non-BPS extremal $\mathrm{BH}$ states. 
In non-BPS cases, ADM mass of extremal BH states is not directly related to the skeweigenvalues of the central charge matrix $Z_{A B}$. For some non-BPS extremal BHs a "fake supergravity (first order) formalism" [55] can be consistently formulated in terms of a "fake superpotential" $\mathcal{W}(\phi, \mathcal{P})$ [56, 57, 58, 59] such that (also recall Eq. [1.5))

$$
\begin{aligned}
\left.\mathcal{W}_{\text {non-BPS }}^{2}(\phi, \mathcal{P})\right|_{\frac{\partial \mathcal{W}}{\partial \phi}=0} \equiv \mathcal{W}_{\text {non-BPS }}^{2}\left(\phi_{H, n o n-B P S}(\mathcal{P}), \mathcal{P}\right)= \\
=\left.V_{B H}(\phi, \mathcal{P})\right|_{\frac{\partial V_{B H}}{\partial \phi}=0} \equiv V_{B H}\left(\phi_{H, n o n-B P S}(\mathcal{P}), \mathcal{P}\right)= \\
=\frac{S_{B H, n o n-B P S}(\mathcal{P})}{\pi},
\end{aligned}
$$

with $\mathcal{W}_{\text {non-BPS }}$ varying, dependently on whether $Z_{A B}=0$ or not. In such frameworks, the general expression of the non-BPS ADM mass reads as follows [56, 57, 58]

$$
M_{A D M, n o n-B P S}\left(\phi_{\infty}, \mathcal{P}\right)=\mathcal{W}_{\text {non-BPS }}\left(\phi_{\infty}, \mathcal{P}\right)
$$

\section{Acknowledgments}

This work is supported in part by the ERC Advanced Grant no. 226455, "Supersymmetry, Quantum Gravity and Gauge Fields" (SUPERFIELDS).

We would like to thank M. Trigiante for enlightening discussions.

A. M. would like to thank the CTP of the University of California, Berkeley, CA USA, the Department of Physics, University of Cincinnati, OH USA, and the Department of Physics, Theory Unit Group at CERN, Geneva $\mathrm{CH}$, where part of this work was done, for kind hospitality and stimulating environment.

The work of B. L. C. and B. Z. has been supported in part by the Director, Office of Science, Office of High Energy and Nuclear Physics, Division of High Energy Physics of the U.S. Department of Energy under Contract No. DE-AC02-05CH11231, and in part by NSF grant 10996-13607-44 PHHXM.

A substantial part of S. F.'s investigation was performed at the Center for Theoretical Physics (CTP), University of California, Berkeley, CA USA, with S. F. sponsored by a "Miller Visiting Professorship" awarded by the Miller Institute for Basic Research on Science. The work of S. F. has been supported also in part by INFN - Frascati National Laboratories, and by D.O.E. grant DE-FG03-91ER40662, Task C.

The work of A. M. has been supported by an INFN visiting Theoretical Fellowship at SITP, Stanford University, Stanford, CA, USA.

\section{References}

[1] M. K. Gaillard and B. Zumino, Duality Rotations for Interacting Fields, Nucl. Phys. B193, 221 (1981). 
[2] S. Ferrara, J. Scherk and B. Zumino, Algebraic Properties of Extended Supergravity Theories, Nucl. Phys. B121, 393 (1977).

[3] S. Ferrara and J. M. Maldacena, Branes, central charges and U duality invariant BPS conditions, Class. Quant. Grav. 15, 749 (1998), hep-th/9706097.

[4] S. Ferrara and M. Günaydin, Orbits of exceptional groups, duality and BPS states in string theory, Int. J. Mod. Phys. A13, 2075 (1998), hep-th/9708025.

[5] H. Lu, C. N. Pope and K. S. Stelle, Multiplet structures of BPS solitons, Class. Quant. Grav. 15, 537 (1998), hep-th/9708109.

[6] S. Bellucci, S. Ferrara, M. Günaydin and A. Marrani, Charge orbits of symmetric special geometries and attractors, Int. J. Mod. Phys. A21, 5043 (2006), hep-th/0606209.

[7] C. Hull and P. K. Townsend, Unity of Superstring Dualities, Nucl. Phys. B438, 109 (1995), hep-th/9410167.

[8] S. W. Hawking, Gravitational Radiation from Colliding Black Holes, Phys. Rev. Lett. 26, 1344 (1971). J. D. Bekenstein, Black Holes and Entropy, Phys. Rev. D7, 2333 (1973).

[9] R. D'Auria, S. Ferrara, M. A. Lledó, On central charges and Hamiltonians for 0-brane dynamics, Phys. Rev. D60, 084007 (1999), hep-th/9903089.

[10] E. Cartan, Euvres complètes (Editions du Centre National de la Recherche Scientifique, Paris, 1984).

[11] E. Cremmer and B. Julia, The SO(8) Supergravity, Nucl. Phys. B159, 141 (1979).

[12] R. Kallosh and B. Kol, E $E_{7(7)}$ symmetric area of the black hole horizon, Phys. Rev. D53, R5344 (1996), hep-th/9602014.

[13] L. Andrianopoli, R. D'Auria, S. Ferrara and M. Trigiante, Extremal black holes in supergravity, Lect. Notes Phys. 737, 661 (2008), hep-th/0611345.

[14] M. Cvetic and D. Youm, Dyonic BPS saturated black holes of heterotic string on a six torus, Phys. Rev. D53, R584 (1996), hep-th/9507090. M. Cvetic and A. A. Tseytlin, General class of BPS saturated dyonic black holes as exact superstring solutions, Phys. Lett. B366, 95 (1996), hep-th/9510097.

[15] M. J. Duff, J. T. Liu and J. Rahmfeld, Four-dimensional string-string-string triality, Nucl. Phys. B459, 125 (1996), hep-th/9508094.

[16] S. Ferrara, R. Kallosh and A. Strominger, $\mathcal{N}=2$ extremal black holes, Phys. Rev. D52, R5412 (1995), hep-th/9508072.

[17] A. Strominger, Macroscopic entropy of $\mathcal{N}=2$ extremal black holes, Phys. Lett. B383, 39 (1996), hep-th/9602111. 
[18] S. Ferrara and R. Kallosh, Supersymmetry and attractors, Phys. Rev. D54, 1514 (1996), hep-th/9602136.

[19] S. Ferrara and R. Kallosh, Universality of supersymmetric attractors, Phys. Rev. D54, 1525 (1996), hep-th/9603090.

[20] S. Ferrara, G. W. Gibbons and R. Kallosh, Black Holes and Critical Points in Moduli Space, Nucl. Phys. B500, 75 (1997), hep-th/9702103.

[21] L. Andrianopoli, R. D'Auria and S. Ferrara, Central extension of extended supergravities in diverse dimensions, Int. J. Mod. Phys. A12, 3759 (1997), hep-th/9608015. L. Andrianopoli, R. D'Auria and S. Ferrara, $U$ duality and central charges in various dimensions revisited, Int. J. Mod. Phys. A13, 431 (1998), hep-th/9612105. L. Andrianopoli, R. D'Auria and S. Ferrara, Flat symplectic bundles of $\mathcal{N}$ extended supergravities, central charges and black hole entropy, lectures given at APCTP Winter School on Dualities of Gauge and String Theories, Seoul and Sokcho, Korea, 17-28 Feb. 1997, hep-th/9707203.

[22] A. Ceresole, R. D'Auria and S. Ferrara, The Symplectic structure of $\mathcal{N}=2$ supergravity and its central extension, talk given at ICTP Trieste Conference on Physical and Mathematical Implications of Mirror Symmetry in String Theory, Trieste, Italy, 5-9 June 1995, Nucl. Phys. Proc. Suppl. 46, 67 (1996), hep-th/9509160.

[23] E. G. Gimon, F. Larsen and J. Simon, Black holes in Supergravity: The Non-BPS branch, JHEP 0801, 040 (2008), arXiv:0710.4967.

[24] V. Balasubramanian, E. G. Gimon and T. S. Levi, Four Dimensional Black Hole Microstates: From D-branes to Spacetime Foam, JHEP 0801, 056 (2008), hep-th/0606118.

[25] F. Denef, D. Gaiotto, A. Strominger, D. Van den Bleeken and X. Yin, Black Hole Deconstruction, hep-th/0703252.

[26] B. de Wit, F. Vanderseypen and A. Van Proeyen, Symmetry structure of special geometries, Nucl. Phys. B400, 463 (1993), hep-th/9210068.

[27] R. Arnowitt, S. Deser and C. W. Misner, Canonical Variables for General Relativity, Phys. Rev. 117, 1595 (1960).

[28] G. W. Gibbons and C. M. Hull, A Bogomol'ny Bound for General Relativity and Solitons in $\mathcal{N}=2$ Supergravity, Phys. Lett. B109, 190 (1982).

[29] L. Andrianopoli, R. D'Auria and S. Ferrara, U-Invariants, Black-Hole Entropy and Fixed Scalars, Phys. Lett. B403, 12 (1997), hep-th/9703156.

[30] S. Ferrara and R. Kallosh, On $\mathcal{N}=8$ Attractors, Phys. Rev. D73, 125005 (2006), hep-th/0603247.

[31] R. Gilmore, Lie Groups, Lie Algebras, and Some of Their Applications (Dover Publications, New York, 2006). 
[32] S. Ferrara and A. Marrani, $\mathcal{N}=8$ non-BPS Attractors, Fixed Scalars and Magic Supergravities, Nucl. Phys. B788, 63 (2008), arXiv:0705.3866.

[33] S. Ferrara and A. Marrani, On the Moduli Space of non-BPS Attractors for $\mathcal{N}=2$ Symmetric Manifolds, Phys. Lett. B652, 111 (2007), arXiv:0706.1667.

[34] S. Bellucci, S. Ferrara, A. Marrani and A. Yeranyan, stu Black Holes Unveiled, Entropy 10(4), 507 (2008), arXiv:0807.3503.

[35] E. Bergshoeff, I. G. Koh and E. Sezgin, Coupling of Yang-Mills to $\mathcal{N}=4, D=4$ Supergravity, Phys. Lett. B155, 71 (1985).

[36] M. de Roo and P. Wagemans, Gauge Matter Coupling In $\mathcal{N}=4$ Supergravity, Nucl. Phys. B262, 644 (1985).

[37] E. Cremmer, S. Ferrara and J. Scherk, SU(4) Invariant Supergravity Theory, Phys. Lett. B74, 61 (1978).

[38] M. J. Duff, J. T. Liu and J. Rahmfeld, Four-dimensional string-string-string triality, Nucl. Phys. B459, 125 (1996), hep-th/9508094; M. Cvetic and A. A. Tseytlin, Solitonic strings and BPS saturated dyonic black holes, Phys. Rev. D53, 5619 (1996); Erratumibid. D55, 3907 (1997), hep-th/9512031.

[39] S. Bellucci, S. Ferrara, R. Kallosh and A. Marrani, Extremal Black Hole and Flux Vacua Attractors, contribution to the Proceedings of the Winter School on Attractor Mechanism 2006 (SAM2006), 20-24 March 2006, INFN-LNF, Frascati, Italy, arXiv:0711.4547.

[40] P. Aschieri, S. Ferrara and B. Zumino, Duality Rotations in Nonlinear Electrodynamics and in Extended Supergravity, arXiv:0807.4039.

[41] B. de Wit, Electric-Magnetic Duality in Supergravity, Nucl. Phys. Proc. Suppl. 101, 154 (2001), hep-th/0103086.

[42] J. F. Luciani: Coupling of O(2) Supergravity with Several Vector Multiplets, Nucl. Phys. B132, 325 (1978).

[43] E. Cremmer and A. Van Proeyen, Classification Of Kahler Manifolds In $\mathcal{N}=2$ Vector Multiplet Supergravity Couplings, Class. Quant. Grav. 2, 445 (1985).

[44] M. Günaydin, G. Sierra and P. K. Townsend, The Geometry of $\mathcal{N}=2$ Maxwell-Einstein Supergravity and Jordan Algebras, Nucl. Phys. B242, 244 (1984).

[45] P. K. Tripathy and S. P. Trivedi, Non-supersymmetric attractors in string theory, JHEP 0603, 022 (2006), hep-th/0511117.

[46] R. D'Auria, S. Ferrara and M. Trigiante, Critical points of the Black-Hole potential for homogeneous special geometries, JHEP 0703, 097 (2007), hep-th/0701090. 
[47] S. Cecotti, Homogeneous Kahler Manifolds And T Algebras In $\mathcal{N}=2$ Supergravity And Superstrings, Commun. Math. Phys. 124, 23 (1989).

[48] M. Günaydin, A. Neitzke, B. Pioline and A. Waldron, Quantum Attractor Flows, JHEP 0709, 056 (2007), arXiv:0707.0267.

[49] D. Gaiotto, W. W. Li and M. Padi, Non-Supersymmetric Attractor Flow in Symmetric Spaces, JHEP 0712, 093 (2007), arXiv:0710.1638.

[50] E. Bergshoeff, W. Chemissany, A. Ploegh, M. Trigiante and T. Van Riet, Generating Geodesic Flows and Supergravity Solutions, arXiv:0806.2310.

[51] L. Andrianopoli, M. Bertolini, A. Ceresole, R. D'Auria, S. Ferrara and P. Fré, General matter coupled $\mathcal{N}=2$ supergravity, Nucl. Phys. B476, 397 (1996), hep-th/9603004. L. Andrianopoli, M. Bertolini, A. Ceresole, R. D'Auria, S. Ferrara , P. Fré and T. Magri, $\mathcal{N}=2$ supergravity and $\mathcal{N}=2$ superYang-Mills theory on general scalar manifolds: Symplectic covariance, gaugings and the momentum map, J. Geom. Phys. 23, 111 (1997), hep-th/9605032.

[52] G. W. Gibbons, M. J. Perry and C. N. Pope, The First law of thermodynamics for Kerr-anti-de Sitter black holes, Class. Quant. Grav. 22, 1503 (2005), hep-th/0408217.

[53] W. Chen, H. Lu and C. N. Pope, Mass of rotating black holes in gauged supergravities, Phys. Rev. D73, 104036 (2006), hep-th/0510081.

[54] S. Ferrara, C. A. Savoy and B. Zumino, General Massive Multiplets In Extended Supersymmetry, Phys. Lett. B100, 393 (1981).

[55] D. Z. Freedman, C. Nunez, M. Schnabl and K. Skenderis, Fake supergravity and domain wall stability, Phys. Rev. D69, 104027 (2004), hep-th/0312055. A. Celi, A. Ceresole, G. Dall'Agata, A. Van Proeyen and M. Zagermann, On the fakeness of fake supergravity, Phys. Rev. D71, 045009 (2005), hep-th/0410126. M. Zagermann, $\mathcal{N}=4$ fake supergravity, Phys. Rev. D71, 125007 (2005), hep-th/0412081. K. Skenderis and P. K. Townsend, Hidden supersymmetry of domain walls and cosmologies, Phys. Rev. Lett. 96, 191301 (2006), hep-th/0602260. D. Bazeia, C.B. Gomes, L. Losano and R. Menezes, First-order formalism and dark energy, Phys. Lett. B633, 415 (2006), astro-ph/0512197. K. Skenderis and P. K. Townsend, PseudoSupersymmetry and the Domain-Wall/Cosmology Correspondence, J. Phys. A40, 6733 (2007), hep-th/0610253.

[56] A. Ceresole and G. Dall'Agata, Flow Equations for Non-BPS Extremal Black Holes, JHEP 0703, 110 (2007), hep-th/0702088.

[57] L. Andrianopoli, R. D'Auria, E. Orazi, M. Trigiante, First Order Description of Black Holes in Moduli Space, JHEP 0711, 032 (2007), arXiV:0706.0712.

[58] S. Ferrara, A. Gnecchi and A. Marrani, $d=4$ Attractors, Effective Horizon Radius and Fake Supergravity, Phys. Rev. D78, 065003 (2008), arXiv:0806.3196. 
[59] J. Perz, P. Smyth, T. Van Riet and B. Vercnocke, First-order flow equations for extremal and non-extremal black holes, arXiv:0810.1528. 